\title{
Relative Performance Evaluation in Presence of Exposure Risk ${ }^{1}$
}

\author{
Robert F. Göx ${ }^{2}$
}

August 2016

${ }^{1}$ The author acknowledges helpful comments and suggestions from Ana Albuquerque, Sanjay Banerjee (MAS discussant), Jeremy Bertomeu, Hui Chen, Thomas Hemmer, Daniel Kauth, Ulrich Schaefer, participants of the 2016 EIASM Workshop on Accounting and Economics, the 2015 AAA MAS midyear meeting in Newport Beach, the EAA 2015 Glasgow, and workshop participants at Columbia University, Baruch College, HEC Lausanne, and UCLA.

${ }^{2}$ Robert F. Göx, University of Zurich, Department of Business Administration, Chair of Managerial Accounting, Seilergraben 53, CH-8001 Zürich, Switzerland. Phone: +41 44634 5965, email: robert.goex@business.uzh.ch. 


\title{
Relative Performance Evaluation in Presence of Exposure Risk
}

\begin{abstract}
I study the consequences of a random exposure to common risk for the purpose of relative performance evaluation (RPE) and find that it significantly affects the usefulness and the empirical measurement of RPE. According to my analysis, the magnitude of the exposure risk not only determines how firms aggregate measures of common risk with measures of firm performance but also the extent to which the firms can control the impact of common risk on their own performance. Simulated regressions of my theoretical model indicate that a high exposure risk can prevent the correct identification of informative performance signals and cause a biased composition of customized peer groups. A high exposure risk also increases the likelihood of a type II error in implicit RPE tests. I evaluate two empirical strategies to control for the magnitude of the exposure risk and find that they significantly reduce the likelihood of a type II error.
\end{abstract}

Keywords: executive compensation, relative performance evaluation, reward for luck, exposure to common risk

JEL Classification: J33, M40, M46 


\section{Introduction}

\subsection{Motivation and Research Question}

I examine the consequences of a random exposure to common risk for the use and empirical measurement of relative performance evaluation (henceforth RPE). Agency theory recommends RPE to shield agents against the compensation risk arising from the volatility of risk factors beyond their control. Considering the agent's action as a random parameter of the underlying performance distribution, an optimal RPE contract aggregates measures of firm performance and common risk so that the combined performance signal is a more informative measure of the agent's action than the raw measure of firm performance. Evaluating the agent on the basis of an optimally aggregated performance measure improves the risk sharing between principal and agent to the benefit of firm owners (Holmstrom 1979, 1982).

Compared to its theoretical benefits, the evidence on the use of RPE is scant. This apparent contradiction between theory and practice has spurred a large body of theoretical and empirical research. In an attempt to solve the so called RPE puzzle (Murphy 1999) theorists have considered various extensions of the basic agency model to rationalize the scarce use of RPE. Different from the present paper, these studies typically consider settings where RPE also serves other purposes than evaluating the agent's performance (e.g. Aggarwal and Samwick 1999, Garvey and Milbourn 2003, Gopalan et al. 2010). At the same time, empiricists have used refined research designs (Albuquerque 2009, 2014) and newly available data (Gong et al. 2011) to shed light on the factors determining the (lack of the) use of RPE. $^{1}$

In this paper, I study the consequences of an important practical difficulty that constrains the implementation of RPE in real world compensation contracts but has so far been overlooked in the academic debate, namely the randomness of firms' exposure to common risk. As I will argue and demonstrate in more detail below, a random exposure to common risk factors limits the ability to remove the impact of these factors from measures of firm performance. The resulting exposure risk not only makes RPE less useful than suggested by the standard agency model but it also impedes the empirical identification of RPE in

\footnotetext{
${ }^{1}$ See section 2 for a detailed review and discussion of the related literature.
} 
cases where firms optimally remove the measurable part of common risk from their own performance.

To illustrate the problem, consider a slightly modified example of an oil producer proposed by Bertrand and Mullainathan (2001) to demonstrate the seemingly obvious benefits of removing the impact of observable random factors from measures of firm performance. The firm's profit is a linear function of the random oil price $\widetilde{p}$, so that $\widetilde{x}=a+c \cdot \widetilde{p}+\widetilde{\epsilon}$, where $a$ is the unobservable contribution of the firm's manager, $\widetilde{\epsilon}$ is the firm's idiosyncratic risk, and $c$ is the marginal exposure of the firm's performance to changes in the oil price. ${ }^{2}$ If $c$ is a known constant, it is easy to construct a net performance measure $\widetilde{y}=\widetilde{x}-c \cdot \widetilde{p}=a+\widetilde{\epsilon}$ that provides the same effort incentives but shields the manager from the risk of oil price changes. Suppose now, that $c$ depends on the firm's production quantity and that the quantity is also random. ${ }^{3}$ Let $\widetilde{c}$ take two values $\widetilde{c} \in\left\{c_{L}, c_{H}\right\}$ with arbitrary positive probabilities. Since the true value of $\widetilde{c}$ is not known at the time the contract is written, the agent's compensation will inevitably depend on the oil price even if the firm evaluates the agent on the basis of the aggregate performance measure $\widetilde{z}=\widetilde{x}-E[\widetilde{c}] \cdot \widetilde{p}$ that removes the expected impact of the oil price from the firm's performance. ${ }^{4}$

\subsection{Research Design and Key Results}

To explore the implications of a random exposure to common risk for the practice and empirical measurement of RPE, I consider the problem of finding linear aggregates of firm performance and informative but uncontrollable measures of common risk. As in Holmstrom (1982), firm performance is a linear function of the agent's effort, common and idiosyncratic risk. However, different from previous literature, the marginal exposure to common risk is not a constant but a random variable. This seemingly marginal change of a standard

\footnotetext{
${ }^{2}$ Throughout the paper a " $\sim$ " indicates a random variable, whereas the same letter without a " $\sim$ " above denotes its realization.

${ }^{3}$ An unknown production quantity is only one of many possible factors that could cause a random exposure to common risk. For example, it is well know from the asset pricing literature that beta factors exhibit a significant level of volatility (e.g. Jagannathan and Wang 1996, Fama and French 1997, or Lewellen and Nagel 2006). See section 3 for a detailed discussion and further examples.

${ }^{4}$ Let $s(\widetilde{z})$ denote the agent's compensation function, since $\widetilde{z}=\widetilde{y}+(\widetilde{c}-E[\widetilde{c}]) \cdot \widetilde{p}$ and $\widetilde{c} \neq E[\widetilde{c}]$, the agent's compensation is a function of $\widetilde{p}$.
} 
assumption in the basic RPE model has important consequences for the aggregation of performance measures.

Even with multivariate normality of all random variables, the standard solution methods for the aggregation problem proposed in the literature cannot be applied because aggregate performance measures no longer satisfy the necessary distributional assumptions. ${ }^{5}$ To deal with this problem, I derive aggregate performance measures as best linear predictors of firm performance given the information contained in the available measures of common risk. This statistical solution approach has several advantages. First, it replicates the relevant results found in the literature for a constant common risk exposure. Second, it provides a clear benchmark for studying the consequences of exposure risk for the empirical measurement of RPE. Third, it can be reconciled with an optimal contracting approach albeit under some restrictive conditions.

Using this methodology, I derive linear aggregation rules for the performance of a focal firm with three different signal structures that cover the common problem settings considered in previous literature. The first case examines the problem of filtering firm performance for an observable factor of common risk in order to avoid reward for luck (henceforth RFL). The second case considers the aggregation of firm performance with a single noisy measure of common risk, and the third case considers the construction of a customized peer group and its weight in the aggregate performance index. Unless stated otherwise, I refer to all of these problems as special cases of RPE. ${ }^{6}$

In all cases, the randomness of the common risk exposure affects the covariance structure of performance measures. Most importantly, it increases the variance of a given performance measure by a constant term that I coin exposure risk. This term is an increasing function of the exposure variance and the variance and expectation of common risk. Since the exposure

\footnotetext{
${ }^{5}$ Prior studies of RPE require that performance measures are either jointly normal or that the joint distribution belongs to a particular class of exponential distributions as specified in Banker and Datar (1989). See section 4 for a detailed discussion of existing solutions to the aggregation problem.

${ }^{6}$ Previous literature distinguishes two forms of control for common risk. If common risk factors are not directly observable but other firms are exposed to the same risks, the literature refers to RPE (Holmstrom 1982). In contrast, if common risk factors are directly measurable, such as the oil price in the introductory example, the literature refers to the lack of RPE as reward for luck (RFL) despite the fact that the failure to implement this recommendation could also penalize agents for bad luck (Bertrand and Mullainathan 2001).
} 
risk contained in a given performance measure cannot be eliminated or reduced by RPE, it adds to the idiosyncratic risk contained in the performance signal and makes it less useful for the control of common risk.

My analysis of aggregation rules yields the following results: First, I find that the random exposure to common risk precludes the perfect filtering of observable random factors. Moreover, since the firm's own exposure risk is not reduced by the use of RPE, the resulting variance reduction is smaller than in the standard case of a constant exposure to common risk. This observation holds for all three signal structures studied in this paper. Second, I find that the optimal weight on a noisy measure of common risk is mainly determined by the exposure risk contained in the performance signal. Third, I find that the relative weights of peer firms within a customized peer index are monotonically decreasing in the peers' exposure risk. I also demonstrate that the weight of a peer index consisting of identical firms as in Holmstrom (1982) is a decreasing function of the standardized exposure risk. Overall, these observations suggest that the presence of exposure risk significantly reduces the relevance of common risk measures for performance evaluation purposes.

To complement my theoretical analysis, I simulate two sets of regression models with randomly generated data for the signal structures used in the theoretical part of the paper. The first set of regressions explores how varying degrees of exposure risk affect the statistical identification of relevant common risk measures. The results suggest that an increasing exposure risk impairs the firms' ability to determine the relevant set of performance measures and peer firms. Moreover, since the weights of individual peer firms are determined simultaneously, the failure to identify a subset of relevant performance peers also biases the weights put on properly identified peers.

In a second set of simulated regressions, I study how the random exposure to common risk could affect the correct empirical identification of RPE. I find that the presence of exposure risk significantly increases the likelihood of a type II error in implicit RPE tests and gives the wrong impression that firm using optimal aggregation rules fail to control for common risk. I examine two potential empirical strategies for addressing this problem. In the first test, I add a dummy variable identifying firms with an exposure risk above the sample mean and find that this proxy significantly improves the correct identification of RPE provided that the measurement of exposure risk is not too noisy. In a second test, I use a random 
coefficient regression model as an indirect control for exposure risk at the firm level and find that this method significantly reduces the likelihood of a type II error even if exposure risk is high. These results suggest that the power of implicit RPE tests could be improved by the use of appropriate controls for the magnitude of exposure risk at the firm level.

The contribution of my analysis to the RPE literature is threefold. First, I identify the size of the exposure risk as a relevant factor for determining the aggregation of performance measures and the composition of peer groups for RPE. Second, I provide a new explanation for the limited use of RPE in the context of a model where its sole purpose is performance evaluation. My findings might help to understand why recent research on the explicit use of relative performance RPE in executive compensation contracts finds that a large majority of firms do not use RPE in executive compensation contracts (Bannister and Newman 2003; Gong et al. 2011). Third, my study provides a new reason for the limited predictive power of implicit RPE tests and suggests two different remedies for this problem.

The rest of the paper is organized as follows. Section 2 provides an overview of the related theoretical literature. Comprehensive summaries of the empirical literature can be found in Albuquerque (2009) and Dikolli et al. (2013). Section 3 explains the model assumptions. Section 4 reviews the existing benchmark solutions for the aggregation problem in the absence of exposure risk and derives the objective criterion for the aggregation of performance measures in presence of exposure risk. Section 5 derives linear aggregation rules in presence of exposure risk for three different signal structures. Section 6 provides simulated regression analyses based on the theoretical model and explores additional implications for empirical research. Section 7 concludes the analysis with a summary of the main findings and some suggestions for future research.

\section{Related Literature}

To my best knowledge, the present paper is the first to analyze the potential consequences of exposure risk on RPE. In the appendix of a recent empirical paper, Albuquerque (2014) sketches a related idea in the context of a LEN model. She assumes that the covariance between firm performance $\widetilde{x}$ and peer performance $\widetilde{y}$ is a random variable that must be estimated from observing a noisy signal $\widetilde{s}_{x y}$ of the true covariance $\widetilde{\sigma}_{x y}$. Without affecting the 
variance of the performance measures, this approach can yield more or less RPE depending on the signal realization $s_{x y}$.

Several other theoretical studies have proposed various theoretical arguments that could explain RFL or the lack and/or limited use of RPE. For example, Aggarwal and Samwick (1999) argue that firms in industries with a small number of competitors have an incentive to put a positive weight on peer performance in order to soften product market competition and show that this incentive can outweigh the potential benefits of RPE. Garvey and Milbourn (2003) consider a setting where RPE takes the form of costly hedging a given market index. In their model, the CEO and the firm can both hedge the market index at different cost. The optimal level of RPE depends on the relative magnitude of hedging costs and decreases if hedging becomes relatively more costly for the firm.

Maug (2000) studies the relation between RPE and the agent's own asset portfolio. He shows that the manager optimally combines the assets in his portfolio so that he minimizes his total compensation risk. Since an optimal RPE contract essentially achieves the same objective in a world where the manager's own trading activities are ignored, RPE becomes redundant if the manager can optimally diversify his compensation risk by own trading activities. Gopalan et al. (2010) study a model where the CEO decides on a firm's exposure to the performance of the industry sector in which it operates. Before deciding on the firm's exposure, the CEO can exert costly effort to acquire a private signal about future sector performance. To motivate the CEO's effort and exposure choices, the optimal contract is positively related to sector performance. Finally, Fershtman et al. (2003) consider a model where the agent derives additional utility from receiving a larger pay package than his peers and find that the agent's social preferences require that the optimal contract exhibits a positive exposure to peer performance.

Other studies provide rational explanations for the presence of RFL in executive compensation contracts as empirically documented in Bertrand and Mullainathan (2001) and Garvey and Milbourn (2006). For example, Göx (2008) shows that RFL can be an optimal response to the million-dollar cap for the tax deductibility of non-performance-based pay in Section 162(m) of the Internal Revenue Code. In a related paper, Ferriozi (2011) studies a binary limited liability model with post-contract-pre-decision information and shows that RFL can improve the agent's implicit incentives to avoid bankruptcy. 
Two recent theoretical studies provide potential explanations for the lack of empirical support found in implicit tests of RPE. Dikolli et al. (2013) study the optimal composition of a peer index in the context of a LEN model. They show that implicit tests of RPE are potentially biased against finding support for the use of RPE if firms and empiricists use different aggregation rules for constructing the peer group. However, the authors only obtain this result if they allow firms to depart from optimal aggregation rules. In a related study, Dikolli et al. (2014) examine the consequences of CEO power on the optimal aggregation of peer groups. Assuming that the CEO can use her power to set the weight on a given peer index, they find that the resulting contract does not completely remove common risk from the agent's compensation. As a consequence, implicit RPE tests are unlikely to find evidence for the optimal use of RPE.

\section{Model}

I consider the relative performance evaluation problem of a representative firm. A risk and effort-averse manager (the agent) runs the firm's operations on behalf of a group of riskneutral firm owners (the principal). As in Holmstrom (1982), firm performance

$$
x_{i}=a_{i}+c_{i} \cdot \eta+\varepsilon_{i},
$$

is a linear function of the agent's effort $a_{i}$ and the realizations of two independent random variables $\widetilde{\eta}$ and $\widetilde{\varepsilon}_{i}$, where $E\left[\widetilde{\varepsilon}_{i}\right]=0$, and $E[\widetilde{\eta}]>0$. There are $n$ firms in the economy with the output structure in (1). In what follows, I use the index $i=0$ for the focal firm to distinguish it from its peers.

The first random variable in (1), $\tilde{\eta}$, is a common risk factor and $c_{i}=\partial x_{i} / \partial \eta$ is the firm's (marginal) exposure to common risk. The common risk factor represents economic variables that affect the performance of all firms in the economy, such as input prices, foreign exchange rates, interest rates, stock market conditions, and other factors determining the state of the economy. The second random variable, $\widetilde{\varepsilon}_{i}$, is idiosyncratic and represents factors that affect the performance of firm $i$ only. Examples for the second factor are the success of the firm's R\&D activities, the efficiency of its production processes, or idiosyncratic shocks in product demand. 
The fundamental difference between the two random factors for the purpose of incentive contracting is the fact that $\widetilde{\varepsilon}_{i}$ is not measurable whereas $\widetilde{\eta}$ can at least be measured with noise. More specifically, I assume that there is a publicly observable signal $\widetilde{y}$ that is informative about the common risk factor $\widetilde{\eta}$ but not controllable by the agent of the focal firm, i.e. the distribution of $\widetilde{y}$ does not depend on $a_{0}$. Since the agent is risk averse, the principal has an interest to shield him from the compensation risk caused by the common risk factor and to evaluate his performance relative to the realization of the informative signal $\widetilde{y}$. As in Holmstrom and Milgrom (1987), I assume that the focal firm evaluates the agent on the basis of a performance index that takes the form of a linear aggregation of realized firm performance and the value of the signal about the common risk factor

$$
z_{0}\left(x_{0}, y\right)=x_{0}-\alpha \cdot y
$$

where $\alpha$ is the weight the focal firm puts on the realization of $\widetilde{y}$.

The key complication that distinguishes the present study from previous RPE models, is the assumption that firm $i$ 's marginal exposure to common risk, $\widetilde{c}_{i}$, is a random factor on its own. This assumption is based on the well documented fact that firms' exposure to common risk appears to vary over time (Engle 2009). For example, the asset pricing literature has found that firms' exposure to market risk as measured by their beta factors exhibits a significant level of volatility (e.g. Jagannathan and Wang 1996, Fama and French 1997, Lewellen and Nagel 2006, Ang and Chen 2007). Other studies find similar patterns for the exposure of U.S. firms to foreign exchange-rate risk (e.g. Allayannis and Ihrig 2001, Francis et al. 2008) or changes in commodity prices. $^{7}$

Assuming a linear profit function, the exposure risk arises quite intuitively from the fact that the firm's profit basically sums up a number of mathematical products of (output and input) prices and quantities. In many cases, prices and quantities are both random and beyond the firm's control, especially if firms act as price takers in their product and input markets. As illustrated in the introductory example of an oil producer, this problem implies that the firm's revenues and cash receipts are primarily driven by the product of random variables. Similar observations can be made for the food processing industry where raw

\footnotetext{
${ }^{7}$ Comprehensive online data on intertemporal correlation patterns of prices for various asset classes including commodities is provided by The V-Lab (vlab.stern.nyu.edu) organized by The Volatility Institute at NYU.
} 
material costs are an important cost factor and a key driver of product contribution margins. Since the demand for food and input prices are both volatile and largely beyond the firms' control, the profit of food and beverage manufacturers is to a large extent determined by the product of random variables. ${ }^{8}$ These examples and the empirical evidence suggest that the presence of exposure risk is rather the norm than the exception.

To allow for the volatility of the firm's exposure to common risk, I model $\widetilde{c}_{i}$ as an independent random variable with $E\left[\widetilde{c}_{i}\right]>0$. Since all random variables considered in this study are mutually independent, the overall performance risk of firm $i$ takes the form (Goodman, 1960)

$$
V A R\left[\widetilde{x}_{i}\right]=V A R\left[\widetilde{\varepsilon}_{i}\right]+E\left[\widetilde{c}_{i}\right]^{2} \cdot V A R[\tilde{\eta}]+V A R\left[\widetilde{c}_{i}\right] \cdot\left(V A R[\tilde{\eta}]+E[\tilde{\eta}]^{2}\right)
$$

The expression in (3) comprises three terms. The first term, $V A R\left[\widetilde{\varepsilon}_{i}\right]$, represents the idiosyncratic part of firm risk. The second term, $E\left[\widetilde{c}_{i}\right]^{2} \cdot V A R[\widetilde{\eta}]$, is the risk caused by the firm's expected exposure to the common risk factor $\widetilde{\eta}$. Finally, the third term in (3),

$$
R_{i}=V A R\left[\widetilde{c}_{i}\right] \cdot\left(V A R[\widetilde{\eta}]+E[\widetilde{\eta}]^{2}\right)
$$

is the part of the overall firm risk caused by the randomness of the firm's exposure to the common risk factor. As indicated in the introduction, I subsequently refer to $R_{i}$ as the exposure risk of firm $i$ to distinguish this part of the firm's performance risk from the risk caused by other factors.

It can be seen from the expression in (4) that the firm's exposure risk is the product of two factors. The first factor, $V A R\left[\widetilde{c}_{i}\right]$, is the variance of the firm's exposure to $\widetilde{\eta}$. The second factor is the second raw moment of $\widetilde{\eta}$, i.e. $E\left[\widetilde{\eta}^{2}\right]=V A R[\widetilde{\eta}]+E[\widetilde{\eta}]^{2}$. Thus, the exposure risk of firm $i$ is not only increasing in the variance of firm $i$ 's own exposure $\widetilde{c}_{i}$ but also in the variance and the expectation of the common risk factor $\widetilde{\eta}$. The higher the value of each of these factors, the higher is the exposure risk of firm $i$. In other words, the volatility of the common risk factor and the volatility of firm $i$ 's exposure to $\widetilde{\eta}$, are mutually reinforcing each other in augmenting its overall exposure risk.

\footnotetext{
${ }^{8}$ As shown by Kerkvliet and Moffet (1991) and further illustrated by Aabo (2015), global manufacturers face similar problems even if currency prices can be hedged since the randomness of sales prices and/or quantities induces a hedge quantity risk that prevents perfect hedging.
} 
To analyze how the presence of exposure risk affects the standard predictions of RPE models, I derive linear aggregation rules for three different signal structures that cover the most common problem settings considered in the literature. The first case assumes that the common risk factor is directly observable, such as foreign exchange rates or commodity prices, so that

$$
y=\eta
$$

The structure in (5) has been widely used as a benchmark model in studies analyzing the phenomenon of RFL (Bertrand and Mullainathan 2001, Garvey and Milbourn 2006). These studies present evidence suggesting that CEO pay is (positively) related to observable random factors beyond the CEO's control. This observation contradicts the recommendation of agency theory to evaluate the performance of the CEO net of the impact of observable random factors. I also consider a second signal structure, where the signal itself is subject to exposure risk and measurement error

$$
y=c_{j} \cdot \eta+\varepsilon_{j}, \quad j \neq 0 .
$$

The structure in (6) allows me to distinguish a firm's own exposure risk $R_{0}$ from the exposure risk $R_{j}$ and the idiosyncratic risk embedded in the performance measure and to study the consequences of each of these risk factors for the composition of the performance index in (2). Finally, I consider a signal structure that has been frequently used in predicting the optimal aggregation of peer groups into a peer index for the purpose of filtering common shocks that affect the performance of all firms in a given peer group (Holmstrom 1982, Dikolli et al. 2013). For this case, $y$ takes the form of a weighted average of realized peer performance

$$
y=\sum_{j=1}^{n} \gamma_{j} \cdot x_{j}
$$

where $\gamma_{j}$ and $x_{j}$ denote the index weight and the performance of peer firm $j$, respectively, and $x_{j}$ is defined in (1). Since $x_{j}$ is prone to measurement error and exposure risk, the structure in (7) can also be interpreted as an extension of (6), i.e. a rule for filtering an index of $n$ imperfectly measurable random factors beyond the agent's control. 


\section{Linear aggregation of performance measures}

To examine how the presence of exposure risk affects the linear aggregation of performance measures, I first review existing solutions to the aggregation problem and the conditions under which they have been derived. Holmstrom and Milgrom (1987) provide a first solution to the aggregation rule in (2). Assuming that $\widetilde{x}_{0}$ and $\widetilde{y}$ are jointly normal, they show that the optimal weight on realized performance $y$ within the performance index $z_{0}$ equals ${ }^{9}$

$$
\alpha=\frac{C O V\left[\widetilde{x}_{0}, \widetilde{y}\right]}{V A R[\widetilde{y}]} .
$$

Bertrand and Mullainathan (2001) use the result in (8) to derive a theoretical prediction for their empirical test of RFL in executive pay. In their model, $x_{0}$ takes the form of (1) and $y=\eta$ as in (5). However, in contrast to the present study, Bertrand and Mullainathan (2001) take $c_{0}$ as a known constant. With these assumptions $C O V\left[\widetilde{x}_{0}, \widetilde{y}\right]=c_{0} \cdot V A R[\widetilde{y}]$ and $\alpha=c_{0}$. It follows that $z_{0}=a_{0}+\varepsilon_{0}$ so that the agent's compensation does not depend on the realized value of the observable random factor $\widetilde{\eta}$.

Holmstrom (1982) first considers the problem of aggregating multiple peers into a performance index of the form given in (7). Assuming that $c_{i}=1$ for all $i$ and that $\widetilde{\eta}$ as well as all idiosyncratic noise terms $\widetilde{\varepsilon}_{j}$ are independent and normally distributed, he derives the optimal aggregation rule

$$
\gamma_{j}=\frac{\tau_{j}}{\sum_{j=1}^{n} \tau_{j}}
$$

where $\tau_{j}=1 / \operatorname{VAR}\left[\widetilde{\varepsilon}_{j}\right]$ is the precision of $\widetilde{\varepsilon}_{j}$. Extending Holmstrom (1979), he shows that the weighted average of peer firms in (9) is a sufficient statistic for the information contained in the output vector $x=\left(x_{0}, \ldots, x_{n}\right)$ with respect to the agent's effort if $a_{0}$ is considered as a random parameter of the firm's output distribution. More recently, Dikolli et al. (2013) study a variation of Holmstrom's RPE model where $c_{i}$ is a firm specific constant and derive the aggregation rule

$$
\gamma_{j}=\frac{c_{j} \cdot \tau_{j}}{\sum_{j=1}^{n} c_{j} \cdot \tau_{j}}
$$

\footnotetext{
${ }^{9}$ Banker and Datar (1989) derive the same result assuming a general agency model and a class of exponential joint density functions $f\left(x_{0}, y ; a_{0}\right)$ with linear likelihood ratios. Among others, this class includes the normal distribution as a special case.
} 
Different from Holmstrom (1982), who finds his result in the context of a general agency model, Dikolli et al. (2013) determine the aggregation rule in (10) in the context of a LEN-model. Despite the differences in the model details, the results in (8), (9), and (10) are all based on the convenient analytical properties of normally distributed (aggregate) performance measures.

Unfortunately, this approach is not fruitful in the presence of exposure risk because the performance measure in (1) contains the product of the two random variables $\widetilde{c}_{i}$ and $\widetilde{\eta}$. This product is not normally distributed even if $\widetilde{c}_{i}$ and $\widetilde{\eta}$ follow a normal distribution. ${ }^{10}$ Therefore, the performance evaluation problem in presence of exposure risk can neither be solved in the context of a LEN-model, nor can it be found by a rearrangement of probability density functions as in Holmstrom (1982).

To deal with this problem, I do not derive the aggregation rule in (2) from an optimal contracting model but from a statistical objective criterion that yields the results in (8), (9), and (10) without requiring normally distributed performance measures. Since $\widetilde{y}$ is not controllable but informative, its sole purpose is to improve the principal's inference drawn from a given realization of $\widetilde{y}$ on the realization of the common risk factor in $\widetilde{x}_{0}$. This statistical measurement problem can be represented in terms of finding the best linear predictor (BLP) for $\widetilde{x}_{0}$ given $\widetilde{y}$. If $\widetilde{y}$ is a scalar, the BLP takes the form

$$
h(\widetilde{y})=\beta_{0}+\beta_{1} \cdot \widetilde{y}
$$

where the parameters $\beta_{0}$ and $\beta_{1}$ minimize the mean square error $E\left[\left(\widetilde{x}_{0}-h(\widetilde{y})\right)^{2}\right]$. The solution of this estimation problem is equivalent to the result of a linear population regression of $\widetilde{x}_{0}$ on $\widetilde{y}$. Moreover, given the linearity of the aggregation rule in (2), the BLP is equivalent to the conditional expectation function $E\left[\widetilde{x}_{0} \mid \widetilde{y}\right]$ (e.g. Goldberger, 1991, ch. 5; Amemiya 1994, ch. 4). The following proposition defines the objective criterion for the case of multiple signals and relates the result to the aggregation rules in (8), (9), and (10).

\footnotetext{
${ }^{10}$ As Craig (1936) and Epstein (1948) show, the product of two normally distributed random variables with mean $\mu_{i}$ and variance $\sigma_{i}^{2}$ takes the form of a Bessel function. As shown by Aroian (1947) and discussed in further detail by Hayya and Ferrara (1972), this function can only be approximated by a normal distribution if the ratios $\mu_{i} / \sigma_{i}$ approach infinity for both random variables. However, in the context of my model, this feature is not a convenient assumption because it implies that the exposure risk is negligible.
} 
Proposition 1: Let $\widetilde{x}_{0}$ be a random variable and $\widetilde{\mathbf{y}}^{\prime}=\left(\widetilde{y}_{1}, \ldots, \widetilde{y}_{n}\right)$ the transpose of a $n \times 1$ random vector with $n \times n$ covariance-matrix $\mathbf{V}$ and $a n \times 1$ covariance vector $\mathbf{d}$, where

$$
\mathbf{V}=\left(\begin{array}{ccc}
V A R\left[\widetilde{y}_{1}\right], & \ldots & \operatorname{COV}\left[\widetilde{y}_{1}, \widetilde{y}_{n}\right] \\
\vdots & \ddots & \vdots \\
\operatorname{COV}\left[\widetilde{y}_{n}, \widetilde{y}_{1}\right] & \ldots & \operatorname{VAR}\left[\widetilde{y}_{n}\right]
\end{array}\right), \mathbf{d}=\left(\begin{array}{c}
\operatorname{COV}\left[\widetilde{x}_{0}, \widetilde{y}_{1}\right] \\
\vdots \\
\operatorname{COV}\left[\widetilde{x}_{0}, \widetilde{y}_{n}\right]
\end{array}\right) .
$$

With this structure, the BLP takes the form $\beta_{0}+\boldsymbol{\beta}^{\prime} \cdot \tilde{\mathbf{y}}$, where $\boldsymbol{\beta}^{\prime}=\left(\beta_{1}, \ldots, \beta_{n}\right)$ is the transpose of a $n \times 1$ parameter vector satisfying

$$
\boldsymbol{\beta}=\mathbf{V}^{-1} \mathbf{d} .
$$

The aggregation rules in (8), (9), and (10) are special cases of (11) Proof: see appendix.

According to proposition 1, the BLP in (11) reproduces the results in (8), (9), and (10). More importantly, since information on the structure of the covariance-matrix $\mathbf{V}$ and the covariance vector $\mathbf{d}$ is sufficient to determine the BLP, a solution to the aggregation problem can also be found if $c_{i}$ is a random variable. This fact is particularly convenient for two reasons: First, it permits to examine how the presence of exposure risk affects the standard rules for the linear aggregation of performance measures. Second, since the BLP can be interpreted as the expected result of a linear population regression, it is an appropriate benchmark for evaluating the consequences of exposure risk for the results of empirical RPE studies. However, the convenient properties of the BLP do not imply that the aggregation rule in (11) is generally part of the optimal solution of the underlying agency problem. Corollary 1 identifies conditions for which the aggregation in (11) is consistent with the optimal solution of the agency model.

Corollary 1: If the focal firm offers the agent a linear compensation contract $s\left(z_{0}\right)=w_{0}+$ $v_{0} \cdot z_{0}$ and the agent has mean variance-preferences $U_{A}=E\left[s\left(\widetilde{z}_{0}\right)\right]-C\left(a_{0}\right)-h(r) \cdot \operatorname{VAR}\left[s\left(\widetilde{z}_{0}\right)\right]$ as well as strictly convex effort cost $C\left(a_{0}\right)$, the aggregation rule in (11) is part of the optimal solution of the agency problem. Proof: see appendix.

Intuitively, corollary 1 relies on the fact that the BLP is found by minimizing the mean square error. As shown in the appendix, this objective criterion can be reformulated in a way that it is equivalent to the minimization of the variance of the performance measure, $V A R\left[\widetilde{z}_{0}\right]$. If the contract is linear and the agent has mean-variance preferences, the optimal aggregation rule serves the same purpose, that is, the optimal weight $\alpha$ in (2) is set so that 
it minimizes the variance of the agent's performance measure for an arbitrary effort level $a_{0}$. Therefore, the BLP optimally solves the agency problem under the conditions given in corollary 1.

\section{$5 \quad$ Linear aggregation with exposure risk}

\subsection{Filtering observable luck}

I begin the analysis of the consequences of exposure risk for the linear aggregation of performance measures with the simplest case in (5), where the realization of the common risk factor is perfectly measurable. As explained in section 4, Bertrand and Mullainathan (2001) employ this signal structure as a theoretical benchmark for their empirical RFL test. With constant exposure to the common risk factor, the optimal aggregation rule sets the signal weight in the performance index equal to the marginal exposure parameter $\left(\alpha=c_{0}\right)$. The corresponding aggregation rule in the presence of exposure risk is given in proposition 2 .

Proposition 2: If the common risk factor is perfectly measurable, the aggregation rule in (11) becomes

$$
\alpha^{*}=E\left[\widetilde{c}_{0}\right]
$$

Proof: Since $\widetilde{c}_{0}$ and $\widetilde{\eta}$ are independent random variables $C O V\left[\widetilde{x}_{0}, \widetilde{y}\right]=E\left[\widetilde{c}_{0}\right] \cdot V A R[\widetilde{\eta}]$ (Bohrnstedt and Goldberger, 1969). With this covariance, the BLP in (11) becomes (12).

Different from the case of a know exposure to common risk, the aggregation rule in (12) removes only the expected marginal exposure to the common risk factor $\widetilde{\eta}$ from the performance measure in (2) even though the realization of $\widetilde{\eta}$ is perfectly observable. This policy has important consequences for the accuracy of filtering performance measures for observable random factors.

Corollary 2: The presence of exposure risk precludes the complete filtering of observable luck. Proof: Substituting for a from (12) into (2) yields the aggregate performance index

$$
z_{0}\left(\alpha^{*}\right)=a_{0}+\varepsilon_{0}+\left(c_{0}-E\left[\widetilde{c}_{0}\right]\right) \cdot \eta
$$

The expression in (13) is a function of $\eta$. 
According to Corollary 2, the aggregate firm performance varies with the realization of the common risk factor $\widetilde{\eta}$ although the firm removes the expected impact of observable luck from the performance index. The aggregate performance measure in (13) is only independent of $\eta$ if the realized exposure $c_{0}$ happens to equal the expected exposure $E\left[\widetilde{c}_{0}\right]$ but this hairline case is a zero probability event ex ante. Thus, depending on the value of the realized marginal exposure $c_{0}$, aggregate firm performance can either be positively $\left(c_{0}>E\left[\widetilde{c}_{0}\right]\right)$ or negatively $\left(c_{0}<E\left[\widetilde{c}_{0}\right]\right)$ related to the realization of the common risk factor $\widetilde{\eta}$.

In any case, the presence of exposure risk excludes a complete removal of the common risk factor from the performance measure in (2). In fact, for a given effort level $a_{0}$, the filtering rule in (12) reduces the total risk of the focal firm's performance measure by the expected contribution of the common risk factor to the firm's overall performance risk,

$$
V A R\left[\widetilde{x}_{0}\right]-V A R\left[\widetilde{z}_{0}\left(\alpha^{*}\right)\right]=E\left[\widetilde{c}_{0}\right]^{2} \cdot V A R[\widetilde{\eta}]
$$

Accordingly, the resulting variance of the aggregate performance measure in (13),

$$
V A R\left[\widetilde{z}_{0}\left(\alpha^{*}\right)\right]=V A R\left[\widetilde{\varepsilon}_{0}\right]+R_{0}
$$

comprises the idiosyncratic performance risk and a second term, $R_{0}=V A R\left[\widetilde{c}_{0}\right] \cdot E\left[\widetilde{\eta}^{2}\right]$, representing the firm's own exposure risk as defined in (4). The expression in (15) indicates that the optimal aggregation rule in (12) prevents the firm from removing its own exposure risk from the agent's performance measure. Thus, the randomness of the firm's exposure to the common risk factor essentially augments the undiversifiable part of the firm's performance risk and thereby makes the performance measure riskier as for the case of a constant exposure to common risk.

\subsection{Aggregating a noisy measure of common risk}

In this subsection, I consider the aggregation rule for the second signal structure in (6), where the common risk is not perfectly measurable. The signal structure in (6) exhibits two different distortions that prevent the firm from perfectly measuring the realized value of the common risk factor $\widetilde{\eta}$. The first distortion is a standard measurement error represented by

the additive noise term $\widetilde{\varepsilon}_{j}$. The second distortion is the random exposure to the common 
risk factor embedded in the performance measure, represented by the random variable $\widetilde{c}_{j}$, where $j \neq 0$. The aggregation rule for this signal structure is given in proposition 3 .

Proposition 3: If the common risk factor is imperfectly measurable, the aggregation rule in (11) becomes

$$
\alpha^{\circ}=\frac{E\left[\widetilde{c}_{0}\right] \cdot E\left[\widetilde{c}_{j}\right] \cdot V A R[\widetilde{\eta}]}{V A R\left[\widetilde{\varepsilon}_{j}\right]+E\left[\widetilde{c}_{j}\right]^{2} \cdot V A R[\widetilde{\eta}]+R_{j}}
$$

where $R_{j}=V A R\left[\widetilde{c}_{j}\right] \cdot\left(V A R[\widetilde{\eta}]+E[\widetilde{\eta}]^{2}\right)$ is the exposure risk embedded in the performance measure as defined in (4). The performance measure weight in (16) is decreasing in $R_{j}$, $V A R\left[\widetilde{c}_{j}\right]$ and $V A R\left[\widetilde{\varepsilon}_{j}\right]$. It is increasing in $V A R[\widetilde{\eta}]$ and $E\left[\widetilde{c}_{0}\right]$. The effect of $E\left[\widetilde{c}_{j}\right]$ is ambiguous. Proof: see appendix.

A comparison of the aggregation rules in (12) and (16) shows that the presence of measurement distortions embedded in the performance signal $\widetilde{y}$ can have a significant impact on the aggregation of the performance index. Since $R_{j}$ as well as the variances of $\widetilde{c}_{j}$ and $\widetilde{\varepsilon}_{j}$ increase the variance of $\widetilde{y}$ without affecting the covariance between $\widetilde{x}_{0}$ and $\widetilde{y}$, the optimal performance measure weight $\alpha^{\circ}$ is monotonically decreasing in $R_{j}, V A R\left[\widetilde{c}_{j}\right]$ and $V A R\left[\widetilde{\varepsilon}_{j}\right]$. Thus, the additional risk factors embedded in the performance measure decrease the precision of $\widetilde{y}$ and thereby reduce the relative usefulness of $\widetilde{y}$ for protecting the agent's compensation against variations of the common risk factor $\widetilde{\eta}$.

For a given variance of the signal $\widetilde{y}$, its optimal weight in the aggregated performance index is increasing in the expected exposure of the focal firm's performance to the common risk factor, $E\left[\widetilde{c}_{0}\right]$. Intuitively, $\alpha^{\circ}$ is positively related to the firm's expected exposure to common risk because an increasing exposure implies a higher covariance between $\widetilde{x}_{0}$ and $\widetilde{\eta}$ and a higher covariance increases the usefulness of the performance signal $\widetilde{y}$ for reducing the risk of the aggregate performance measure.

Unlike the other factors, a higher variance of the common risk factor $\widetilde{\eta}$ and an increase of $E\left[\widetilde{c}_{j}\right]$ positively affect $C O V\left[\widetilde{x}_{0}, \widetilde{y}\right]$ and $V A R[\widetilde{y}]$. However, an increase of $V A R[\widetilde{\eta}]$ has a positive net effect on $\alpha^{\circ}$ because for any given level of the ratio between the two measures the relative increase of the numerator exceeds the relative change of the denominator. ${ }^{11}$ By contrast, an increase of the signal's expected exposure to the common risk factor, $E\left[\widetilde{c}_{j}\right]$, can

\footnotetext{
${ }^{11}$ In fact, it holds that $\frac{\partial C O V\left[\widetilde{x}_{0}, \widetilde{y}\right]}{\partial V A R[\widetilde{\eta}]} \cdot C O V\left[\widetilde{x}_{0}, \widetilde{y}\right]^{-1}>\frac{\partial V A R(\widetilde{y})}{\partial V A R[\widetilde{\eta}]} \cdot V A R(\widetilde{y})^{-1}$.
} 
reduce or increase $\alpha^{\circ}$. The effect is positive, whenever

$$
V_{j}=V A R\left[\widetilde{\varepsilon}_{j}\right]+R_{j}>E\left[\widetilde{c}_{j}\right]^{2} \cdot V A R[\widetilde{\eta}]
$$

where $V_{j}$ is the total error of $\widetilde{y}$ in measuring $\widetilde{\eta}$. It comprises the idiosyncratic risk $V A R\left[\widetilde{\varepsilon}_{j}\right]$ and the exposure risk $R_{j}$ contained in $\widetilde{y}$. That is, whenever the expected contribution of the common risk factor to the variance of $\widetilde{y}$ on the right hand side of (17) is smaller (larger) than the total measurement error $V_{j}$, an increase of $E\left[\widetilde{c}_{j}\right]$ has a positive (negative) impact on $\alpha^{\circ}$.

Finally, it can be seen that the firm's own exposure risk, $R_{0}$, or equivalently $V A R\left[\widetilde{c}_{0}\right]$, does not affect the aggregation rules in (12) and (16) because it is idiosyncratic to the focal firm and independent of the weight placed on the performance measure $\widetilde{y}$. Corollary 3 summarizes the overall effect of the aggregation rule in (16) on the risk of the aggregated performance measure $\widetilde{z}_{0}\left(\alpha^{\circ}\right)$.

Corollary 3: If the common risk factor is imperfectly measurable, the optimal aggregation rule reduces the risk of the performance measure by

$$
V A R\left[\widetilde{x}_{0}\right]-V A R\left[\widetilde{z}_{0}\left(\alpha^{\circ}\right)\right]=E\left[\widetilde{c}_{0}\right]^{2} \cdot V A R[\widetilde{\eta}] \cdot q^{\circ}, \quad q^{\circ}=\frac{E\left[\widetilde{c}_{j}\right]^{2} \cdot V A R[\widetilde{\eta}]}{V A R[\widetilde{y}]}<1 .
$$

The risk reduction is strictly smaller than the risk reduction with a perfectly measurable common risk factor in (14) and monotonically decreasing in $V A R[\widetilde{y}]$. Proof: Follows from the fact that $V A R[\widetilde{y}]=V_{j}+E\left[\widetilde{c}_{j}\right]^{2} \cdot V A R[\widetilde{\eta}]$ and $\partial q^{\circ} / \partial V A R[\widetilde{y}]<0$.

According to corollary 3, the presence of exposure risk and measurement error in the performance signal $\widetilde{y}$ lowers its usefulness for reducing the variance of the performance index and thereby its desirability for contracting. The higher the exposure risk and the higher the measurement noise embedded in the performance signal, the lower the scaling factor $q^{\circ}$ in (18) that determines the level of risk reduction relative to the benchmark case of a perfectly measurable common risk factor in (14). The scaling factor $q^{\circ}$ has an intuitive interpretation. It is defined as the ratio of the expected amount of common risk to the total risk contained in the signal $\widetilde{y}$ and represents the expected percentage of common risk contained in the performance measure. As shown in (3), $V A R[\widetilde{y}]$ is the sum of three factors: the expected common risk, the idiosyncratic risk, and the exposure risk. Therefore, the scaling factor $q^{\circ}$ is increasing in the expected amount of common risk and decreasing in the amounts of 
idiosyncratic and exposure risk. The consequences of the last observation are summarized below:

Corollary 4: If the exposure risk becomes very large, the signal weight $\alpha^{\circ}$ in (17) and the scaling factor $q^{\circ}$ in (18) both go to zero. Proof: It holds that $\lim _{R_{j} \rightarrow \infty} \alpha^{\circ}=\lim _{R_{j} \rightarrow \infty} q^{\circ}=0$.

Thus, at the limit a substantial exposure risk can render the performance signal $\widetilde{y}$ useless for shielding the agent's compensation against common risk.

\subsection{Aggregation of a peer index}

The last case of my analysis considers the aggregation rule for the third signal structure in (7) where the performance index $\widetilde{y}$ comprises a group of peer firms with a similar performance structure. That is, the performance of each of the $n$ potential peer firms is affected by an idiosyncratic shock $\widetilde{\varepsilon}_{j}$ and each peer faces a random exposure to the common risk factor $\widetilde{\eta}$ represented by the random variable $\widetilde{c}_{j}$. The optimal composition of the peer index in (7) is summarized in proposition 4.

Proposition 4: If the performance index comprises a weighted average of peer firms with firm performance $\widetilde{x}_{j}$ as defined in (1), the optimal weight of firm $j$ equals

$$
\gamma_{j}^{+}=\frac{E\left[\widetilde{c}_{j}\right] \cdot \delta_{j}\left(R_{j}\right)}{\sum_{j=1}^{n} E\left[\widetilde{c}_{j}\right] \cdot \delta_{j}\left(R_{j}\right)}
$$

where $\delta_{j}\left(R_{j}\right)=1 / V_{j}$ is the total precision of the peer signal $\widetilde{x}_{j}$ in measuring $\widetilde{\eta}$ and $V_{j}=$ $\operatorname{VAR}\left(\widetilde{\varepsilon}_{j}\right)+R_{j}$ as defined in (17).

The following observations can be made:

1) The optimal peer weight in (19) is increasing in $E\left[\widetilde{c}_{j}\right]$ and $\delta_{j}\left(R_{j}\right)$, but it is decreasing in $E\left[\widetilde{c}_{k}\right]$ and $\delta_{k}\left(R_{k}\right)$ for $k \neq j$.

2) The optimal weight of peer $j$ is monotonically decreasing in its own exposure risk. It approaches zero as $R_{j}$ approaches infinity.

Proof: see appendix.

A comparison of the aggregation rules in the absence and the presence of exposure risk in (10) and (19) shows that in both cases the index weight of peer firm $j$ is increasing in its (expected) exposure and in its precision in measuring the peer's exposure to the common

risk factor $\widetilde{\eta}$. However, since the signal precision in the presence of exposure risk, $\delta_{j}\left(R_{j}\right)$, 
is decreasing in $R_{j}$, a higher exposure risk reduces the usefulness of firm $j$ for the purpose of RPE. At the limit, if the exposure risk of firm $j$ becomes very large, the firm's optimal weight in the peer index goes to zero. Likewise, for a given exposure and precision of peer firm $j$, an increase of the exposure and/or precision of other firms within the peer index reduces the optimal weight of firm $j$. The opposite is true for an increase in the exposure risk of firm $k$. Because $\delta_{k}\left(R_{k}\right)$ is decreasing in $R_{k}$, a higher exposure risk of firm $k$ increases the optimal weight of all other firms in the performance index.

These considerations show that the exposure risk of peer firms is a critical factor in determining their usefulness for the purpose of relative performance evaluation. The higher the exposure risk of a potential peer firm, the lower its relevance for RPE and for the composition of the peer index. Moreover, since the optimal index weight of each peer firm depends on its own exposure risk as well as on the exposure risk of all other firms contained in the performance index, a change of the exposure risk at one firm affects the optimal index weights of all other firms. Corollary 5 summarizes the impact of exposure risk on the optimal weight of the peer group in the aggregate performance index and the variance of the performance measure.

Corollary 5: With the peer index in (7) and the peer weights in (19), the optimal index weight becomes

$$
\alpha^{+}=\frac{E\left[\widetilde{c}_{0}\right] \cdot V A R[\widetilde{\eta}] \cdot \sum_{j=1}^{n} E\left[\widetilde{c}_{j}\right] \cdot \delta_{j}\left(R_{j}\right)}{1+V A R[\widetilde{\eta}] \cdot \sum_{j=1}^{n} E\left[\widetilde{c}_{j}\right]^{2} \cdot \delta_{j}\left(R_{j}\right)} .
$$

Using the index weight in (20) reduces the risk of the focal firm's performance measure by

$$
V A R\left[\widetilde{x}_{0}\right]-V A R\left[\widetilde{z}_{0}\left(\alpha^{+}\right)\right]=E\left[\widetilde{c}_{0}\right]^{2} \cdot V A R[\widetilde{\eta}] \cdot q^{+}, \quad q^{+}=\frac{z^{+}}{1+z^{+}}<1
$$

where $z^{+}=V A R[\widetilde{\eta}] \cdot \sum_{j=1}^{n} E\left[\widetilde{c}_{j}\right]^{2} \cdot \delta_{j}\left(R_{j}\right)$. The risk reduction is proportional to the scaling factor $q^{+} \in\left(q^{\circ}, 1\right)$ and monotonically increasing in $z^{+}$. Proof: see appendix.

A comparison of the risk reductions achieved with a perfectly measurable common risk factor and the peer index in (14) and (21) shows that the exposure risk contained in the measure of peer performance significantly affects the usefulness of the peer index in reducing the focal firm's compensation risk. Similar to $q^{\circ}$ in (18), the scaling factor $q^{+}$determines the level of risk reduction relative to the benchmark case of a perfectly measurable common risk factor in (14). Its size is determined by the factor $z^{+}$representing a weighted sum of the expected common risks contained in the performance measures of the firms within the 
peer index. Using the definition of $\delta_{j}\left(R_{j}\right)$, the contribution of firm $j$ to the factor $z^{+}$can be expressed as:

$$
z_{j}^{+}=\frac{V A R[\widetilde{\eta}] \cdot E\left[\widetilde{c}_{j}\right]^{2}}{V A R\left[\widetilde{\varepsilon}_{j}\right]+R_{j}},
$$

where $z^{+}=\sum_{j=1}^{n} z_{j}^{+}$. Thus, $z_{j}^{+}$is the ratio of the expected common risk contained in $\widetilde{x}_{j}$ to the sum of the idiosyncractic risk and the exposure risk in $\widetilde{x}_{j}$. The higher the expected common risk and the lower the idiosyncratic risk and the exposure risk, the higher is the incremental contribution of peer firm $j$ to the scaling factor $z^{+}$and thereby to the overall reduction of the performance measure risk. Intuitively, a large expected exposure to common risk increases the usefulness of firm $j$ for RPE, whereas a high exposure risk reduces its desirability for RPE.

To gain additional insights on the impact of the aggregate exposure risk on the optimal level of RPE, assume that all peer firms face identically distributed risk factors. With $\widetilde{\varepsilon}_{j}=\widetilde{\varepsilon}, \widetilde{c}_{j}=\widetilde{c}$, and $R_{j}=R$, the optimal index weight in (20) becomes

$$
\alpha^{+}=\frac{n \cdot E\left[\widetilde{c}_{0}\right] \cdot E[\widetilde{c}] \cdot V A R[\widetilde{\eta}]}{V A R[\widetilde{\varepsilon}]+n \cdot E[\widetilde{c}]^{2} \cdot V A R[\widetilde{\eta}]+R} .
$$

A closer inspection of the expression in (22) shows two facts. First, for a given value of the standardized exposure risk, $R=V A R[\widetilde{c}] \cdot E\left[\widetilde{\eta}^{2}\right]$, the usefulness of RPE is increasing in the number of peer firms because $\partial \alpha^{+} / \partial n>0$. Second, and more important, the standardized exposure risk has a negative impact on the optimal weight of the performance index. The second relation implies the following observation:

Corollary 6: If $R$ approaches infinity, the optimal index weight in (22) approaches zero. Proof: Evident from the structure of the expression in (22).

Thus, at an aggregate level, a high exposure risk can preclude the use of peer groups for the purpose of relative performance evaluation.

\section{Practical and empirical implications of exposure risk}

\subsection{Overview}

The results of the theoretical analysis in section 5 indicate that the presence of exposure risk can have a significant impact on the linear aggregation of performance measures. To illustrate 
the potential economic consequences of varying degrees of exposure risk and to provide additional insights regarding the practical implementation of the theoretical solutions and their implications for empirical research, this section provides two sets of simulation studies. Section 6.2 shows a comprehensive set of firm level simulations for the three different types

of performance measures analyzed in section 5. Section 6.3 extends the focus of the study beyond the firm level and demonstrates how different econometric designs affect the empirical identification of optimal aggregation policies in a heterogeneous firm sample.

\subsection{Firm level analysis}

To illustrate how varying degrees of exposure risk affect a firm's ability to filter observable luck from its own performance, I first compare the results of six different regressions of firm performance $x_{0}$ on common risk $\eta$. Holding all else constant, each regression is performed on 1,000 random samples of 1,000 observations using parameters drawn from six different distributions of the firm's exposure to the common risk factor $\left(\widetilde{c}_{0}\right)$. To isolate the consequences of an increasing exposure risk from changes in other variables, I distinguish the distributions by varying the standard deviation $\sigma_{0}$ from 0 (i.e. a constant exposure) to 12 as shown in panel A of table 1 .

[please insert table 1 about here]

Panel B shows the results of linear regressions of $x_{0}$ on $\eta$ for each of the six exposure distributions shown in panel A. The first four rows report the summary statistics of the estimated regression coefficients of $\eta$. In line with equation (12), the mean estimates are close to $E\left[\widetilde{c}_{0}\right]=1$ for all 6 cases. However, as indicated by the standard deviations and the range of the estimated regression coefficients, exposure risk introduces a substantial degree of variation among the regression coefficients estimated for different random samples.

More importantly, the distribution of $t$-values in rows 5 and 6 as well as the share of significant regression coefficients in row 7 indicate that a high exposure risk decreases the likelihood that a regression finds a statistically significant relation between firm performance and common risk although both variables are correlated. In fact, for a constant exposure (model 1) and a moderate exposure risk (model 2) the regression coefficients are found to be 
significant at the $95 \%$ level for all random samples, whereas only $46.6 \%$ of the estimates for the highest exposure risk (model 6) yield regression coefficients that are significant at the $95 \%$ level. The summary statistics of $R^{2}$ in rows 7 and 8 of panel B with mean values ranging from 0.4997 (case 1) to 0.0055 (case 6) indicate that the variation in firm performance that can be attributed to changes of the common risk factor is decreasing in the firm's exposure risk.

As a practical matter, these findings indicate that a high exposure risk might prevent firms from filtering observable luck from their own performance. In the absence of precise information on the theoretical distributions of $\widetilde{x}_{0}$ on $\widetilde{\eta}$, firms must assess the potential relation between firm performance and observable measures of common risk by means of a regression model similar to the model used in the simulation. Accordingly, the presence of exposure risk impedes the identification of a statistically significant relation between firm performance and common risk. Likewise, exposure risk can render the filtering of observable luck economically insignificant because a low $R^{2}$ indicates a negligible reduction of the firm's overall performance risk.

Panel C provides the summary statistics of an additional test for the efficiency of the optimal filtering rule proposed in equation (12). The test reports simulated regressions for the aggregated performance measure $z_{0}\left(\alpha^{*}\right)$ in equation (13) on common risk $\eta$ using the data generated for the first test in panel B. The results indicate that exposure risk increases the likelihood of finding a significant statistical relation between $z_{0}\left(\alpha^{*}\right)$ and $\eta$ even though the expected impact of common risk has been removed from the firm's performance measure. The shares of significant regression coefficients at the $95 \%$ level with exposure risk are in the range between $14.3 \%$ (model 2) and to $18.2 \%$ (model 6) and significantly higher than in the constant exposure case (model 1) with $5.7 \%$. These findings suggest that the presence of exposure risk increases substantially the likelihood of a type II error (a false rejection of the hypothesis that the firm optimally filters for observable luck) in a compensation study performed at the firm-level and lead to the wrong conclusion that the firm rewards their executives for observable luck. ${ }^{12}$

[please insert table 2 about here]

\footnotetext{
${ }^{12}$ In fact, if the agent's compensation $s(\cdot)$ is a linear function of $z_{0}\left(\alpha^{*}\right)$, a regression of $s(\cdot)$ on $\eta$ will find evidence for RFL.
} 
Table 2 shows equivalent simulation results for the problem of aggregating firm performance and a noisy measure of common risk as studied in section 5.2. The simulation compares the results of six different regressions of firm performance $x_{0}$ on a given measure $y$ of common risk $\eta$. Since the optimal aggregation rule in (16) is not affected by the focal firm's own exposure risk but by the exposure risk contained in the performance signal $y$ (henceforth "index exposure risk"), the standard deviation of $\widetilde{c}_{0}$ is held constant at $\sigma_{0}=1$. To study the consequences of varying index exposure risk, I use the same simulation approach as in table 1 but allow the standard deviation of the index exposure, $\sigma_{1}$, to take 6 different values ranging from 0 to 12 .

The results of the second simulation are in line with those of the first in table 1 . However, since the optimal performance measure weight is negatively related to index exposure risk, the mean values of the regression coefficients are decreasing in $\sigma_{1}$. All other results are similar to those found for varying degrees of the focal firm's own exposure risk in panels B and C of table 1. A higher index exposure risk increases the standard deviation and the range of the regression coefficients relative to the mean and thereby reduces the mean of the $t$-values. As a consequence, the share of regression coefficients that are significant at the $95 \%$ level drops from $100 \%$ in the absence of index exposure risk (model 1) to $26.2 \%$ for the highest index exposure risk (model 6). The same trend can be observed for the distribution of the $R^{2}$-values ranging from 0.1271 (model 1) to 0.0029 (model 6).

The additional regression of the aggregate performance measure $z_{0}\left(\alpha^{\circ}\right)$ on $y$ reported in panel $\mathrm{C}$ of table 2, yields similar results as the regression of $z_{0}\left(\alpha^{*}\right)$ and $\eta$ reported in panel $\mathrm{C}$ of table 1. An overall comparison of tables 1 and 2 suggests that, ceteris paribus, the consequences of an increasing index exposure risk are similar to those of an increase in the focal firm's own exposure risk but most effects found in table 2 are slightly more pronounced than those reported in table 1.

[please insert tables 3 and 4 about here]

Tables 3 an 4 show a numerical example and related simulation results to study the consequences of varying peer exposure risk for the aggregation of a peer index as analyzed in section 5.3. Holding all other parameters constant, the example considers a sample of ten potential peers with identically structured performance signals $x_{j}$ but different degrees 
of exposure risk. The exposure risk of peer $j, j=1, \ldots 10$, is determined by the standard deviation $\sigma_{j}$ of its marginal exposure to common risk, $\widetilde{c}_{j}$. To simplify notation, I let $\sigma_{j}=j$.

As a benchmark for the simulation reported in table 4, table 3 summarizes the theoretically optimal weights for the ten peer firms, $\gamma_{j}^{+}$, and the index, $\alpha^{+}$, as defined in equations (19) and (20), and compares the results to the optimal weights in the absence of exposure risk. The optimal peer weights reported in the second row of table 3 illustrate the importance of exposure risk for the composition of the peer index. The lower the peer exposure risk, the higher the relative weight of a given peer firm within the aggregate performance index. While the performance of the lowest exposure risk firm enters the peer group with a weight of $56.53 \%$, the firm with the highest exposure risk has a weight of $0.84 \%$ only. Multiplying the relative weights with the optimal index weight of 0.3709 yields the effective weight that the focal firm puts on an individual peer firm relative to its own performance.

The comparison of the optimal weights with and without exposure risk in table 3 exhibits significant differences for both the relative weights of individual peers and the aggregate peer index. While the relative weights of individual peer firms are higher (lower) for peers with low (high) exposure risk, the optimal index weight unambiguously declines by $49 \%$ from 0.7273 to 0.3709 due to the presence of exposure risk. Taken together, the example firm optimally puts less effective weights on all firms except for the firm with the lowest exposure risk for which the effective weight rises from 0.0727 to 0.2097 . Overall, the comparison of RPE policies in table 3 indicates that ignoring the presence of peer exposure risk can cause a significant deviation from optimal aggregation rules.

Table 4 shows the summary statistics of 1,000 multiple regressions for simulated random samples of focal firm and peer performance using the parameters of the example in table 3. The mean values of the regression coefficients in the first row of table 4 are close to the effective peer weights reported in row 4 of table 3 . However, as for a given peer index in table 2, a higher peer exposure risk increases the standard deviation and the range of the regression coefficients relative to their mean. As a consequence, the mean $t$-values are significantly declining as the peer exposure risk increases. Likewise, the share of regression coefficients that are significant at the $95 \%$ level drops from $100 \%$ for the lowest peer exposure risk (firm 1) to $20.4 \%$ for the highest peer exposure risk (firm 10). 
These findings suggest that a high exposure risk can restrain firms' ability to efficiently identify and compose optimal peer groups for the purpose of RPE. If precise information on the theoretical distributions of $\widetilde{x}_{0}$ on $\widetilde{x}_{j}$ is lacking, firms need to assess the potential relation between their own performance and the performance of potential peers by means of a regression analysis similar to the model used in table 4 . The low number of significant regression coefficients for peer firms with a sufficiently high exposure risk indicates that this procedure bears the risk of failing to identify the relevant set of peer firms. Moreover, since the weights of individual peer firms are determined simultaneously, the failure to identify a subset of relevant performance peers will necessarily bias the weights put on the peer firms that are properly identified.

\subsection{Firm sample analysis}

The firm level regressions in panel $\mathrm{C}$ of table 1 and table 2 indicate that the presence of exposure risk can cause a type II error in RPE tests performed at the firm-level and lead to the wrong conclusion that firms reward executives for observable luck despite the fact that they actually use the appropriate filtering rules defined in (12) or (16), respectively. To see if the observations made at the firm level can be confirmed for a larger firm sample, as it is typically considered in empirical tests for the use of RPE, I repeat the test from panel $\mathrm{C}$ of table 1 with a simulated data set of 100 firms and 1,000 observations per firm.

[please insert table 5 about here]

To isolate the consequences of a varying exposure risk from other economic variables, all sample firms have the same performance structure and apply the optimal filtering rule defined in (12), but they differ in the variance of their exposure to common risk. Panel A of table 5 shows the distribution of $\sigma_{i}$, the standard deviation of firm $i$ 's exposure to common risk. I consider four different scenarios. The first scenario serves as a benchmark case and considers a constant exposure to common risk $\left(\sigma_{i}=0\right)$. In the other three scenarios, $\sigma_{i}$ is drawn once at the beginning of the simulation study from the uniform distributions given in the first row of panel $\mathrm{A}$. In a second step, the firm specific value of $\sigma_{i}$ is used to generate the firms' period $t$ exposure to common risk by a draw from a normally distributed random variable with $E\left[\widetilde{c}_{i t}\right]=1$ and $V A R\left[\widetilde{c}_{i t}\right]=\sigma_{i}^{2}$. 
Panel B of table 5 reports the results from 1,000 simulated pooled regressions of the optimally aggregated performance measure $z_{i t}=x_{i t}-E\left[\widetilde{c}_{i t}\right] \cdot \eta_{t}$ on the common risk factor $\eta_{t}$ for the randomly generated firm sample. The results are consistent with the firm level analysis and indicate that an increasing level of exposure risk in the firm sample increases the likelihood of a type II error in an empirical RPE test. In the simulation study, the fraction of firms that appear to reward their executives for luck despite the fact that all firms removed the expected contribution of the common risk factor from their performance measure, ranges between $9.3 \%$ for the lowest exposure risk (case 2) and $13.9 \%$ for the highest exposure risk considered in the simulation (case 4). As in the firm level analysis, these values are significantly higher than for a constant exposure (case 1) with a 5.8\% share of significant regression coefficients at the $95 \%$ level.

These results raise the question of whether and how empirical RPE studies could potentially exploit the firm level differences in the variance of the exposure to common risk in order to reduce the likelihood of a type II error. In this study, I consider two options for achieving this objective and evaluate their effectiveness by additional simulation analyses. The first option is the use of a proxy for the firm-specific exposure risk in the regression equation. Provided that the distribution of the firms' exposure to common risk is constant over time, such a measure could be derived from a sequence of firm-level regressions of realized firm performance $x_{i t}$ on the common risk factor $\eta_{t}$ for different time windows. The second option is the use of a regression method that accounts for the heterogeneity in the randomness of the firm's exposure to common risk. The method of choice is a random coefficients model because it explicitly estimates firm specific regression coefficients and thereby allows to control for firm-specific differences in the volatility of the exposure to common risk. ${ }^{13}$

[please insert table 6 about here]

Table 6 presents the results of the first test. To construct an informative measure of the firm-specific exposures risk, I first draw the standard deviation of each firm's exposure risk from a uniform distribution on $[0,2]$ as in the intermediate case 3 of table 5 . In a second

\footnotetext{
${ }^{13}$ Random coefficients models are rarely used in empirical compensation research, an exception is Hermalin and Wallace (2001). Most RPE studies use pooled or firm-fixed effects regression models. In the current setting, both estimation methods are supposed to yield the same results because $\widetilde{c}_{i t}$ and $\widetilde{\eta}_{t}$ are independent (Greene 2008, p.183).
} 
step, I generate a noisy signal $\widetilde{y}_{i}$ of $\sigma_{i}$ by adding a normally distributed noise term $\widetilde{\epsilon}$ with zero mean and $V A R[\widetilde{\epsilon}]=\sigma_{\epsilon}^{2}$. Third, I construct a dummy variable to control for a high exposure risk in the regression equation. The dummy takes the value of 1 if the realized signal value $y_{i}$ is above the sample mean of all firms' signal realizations.

To allow for different signal qualities, I analyze four different cases by gradually varying the standard deviation of the noise term $\widetilde{\epsilon}$ between 0 and 3 as shown in panel A of table 6. Panel B summarizes the main results from 1,000 pooled regressions with a proxy for the magnitude of the firm's exposure risk. The key statistic for determining the likelihood of a type II error is the percentage of significant regression coefficients at the $95 \%$ level for the common risk factor $\left(\widehat{b}_{1}\right)$. With shares of $1.1 \%$ for case $1\left(\sigma_{\epsilon}=0\right)$ and $6.9 \%$ for case 2 $\left(\sigma_{\epsilon}=1\right)$, these shares are significantly lower than the share of $12.7 \%$ found in the relevant case 3 of the univariate regression in table 5 .

These findings indicate that a sufficiently precise proxy variable for the magnitude of the firms' exposure risk can help to reduce the likelihood of a type II error in an empirical RPE test. It can also bee seen that the share of significant regression coefficients for the interaction term $\left(\widehat{b}_{2}\right)$ is of the same order of magnitude as in the univariate regression regardless of the signal precision. This observation shows that the likelihood of the type II error is mainly driven by firms with an exposure risk above the sample average.

[please insert table 7 about here]

Table 7 shows the results of the second test. The setup and the data used for the simulation are the same as for the pooled regression in table 5. Panel B reports the distribution of the regression coefficients found in the simulation of the random coefficients model. While the distribution of the regression coefficients is similar to the results found in the pooled regression in table 5 , the share of significant regression coefficients at the $95 \%$ level is significantly lower than the lowest share of $5.8 \%$ found in the pooled regression model for a constant exposure to the common risk factor. Moreover, the share of significant regression coefficients takes values between $0.5 \%$ and $0.3 \%$ regardless of the magnitude of the exposure risk found in the firm sample. These results indicate that the random coefficients model is an appropriate method for reducing the likelihood of a type II error even for significant levels of exposure risk because it decomposes the regression coefficients into a general and a firm- 
specific component and thereby provides an indirect control for the firm-specific variations of the exposure to common risk.

\section{Summary and suggestions for future research}

I study the consequences of a random exposure to common risk for the purpose of relative performance evaluation (RPE). I find that a random exposure to commons risk can have important consequences for the usefulness and the empirical measurement of RPE. On the one hand, the magnitude of the exposure risk determines how firms aggregate measures of common risk with measures of firm performance. On the other hand, the exposure risk contained in measures of common risk determines the effectiveness of these measures in controlling the impact of common risk on measures of firm performance. As a consequence, the random exposure to common risk can significantly limit the usefulness of RPE.

To explore how varying degrees of exposure risk affect the statistical identification of relevant common risk measures, I complement my theoretical analysis by two sets of regression models with randomly generated observations. In the first set of regressions, I find that an increasing exposure risk impairs the firms' ability to determine the relevant set of performance measures and peer firms. Since the weights of individual peer firms are determined simultaneously, the failure to identify a subset of relevant performance peers also biases the weights put on properly identified peers.

In a second set of simulated regressions, I examine how the random exposure to common risk affects the empirical identification of RPE. I find that a high exposure risk increases the likelihood of a type II error in implicit RPE tests. To address this problem, I examine two empirical strategies to control for the magnitude of the exposure risk. In the first test, I use a dummy variable identifying firms with a high exposure risk. In the second test, I use a random coefficient regression model as an indirect control for exposure risk. While the second method significantly reduces the likelihood of a type II error even for a high exposure risk, the first method performs well only if the firm level measure of exposure risk is not too noisy. These results suggest that the power of implicit RPE tests could be improved by the use of appropriate controls for the magnitude of exposure risk at the firm level. Even though the SEC's new disclosure requirements now permit the use of disclosed peers firms in direct 
tests of RPE (Gong et al. 2011), the refinement of indirect test methods is still a relevant issue for empirical research that seeks to study peer weights or the exposure to non-disclosed common risk factors.

Overall, my analysis suggests that the randomness of firms' exposure to common risk can be an important factor in determining whether or not and how firms practice RPE. Future empirical studies could measure the exposure risk implied by various risk factors and analyze its importance for the explicit use of RPE, the choice of peer firms or changes of peer groups over time and thereby provide evidence for the empirical relevance of exposure risk for the practice of RPE.

\section{Appendix}

\section{Proof of Proposition 1}

Part 1: The BLP is found by minimizing the mean squared error

$$
M S E=E\left[\left(\widetilde{x}_{0}-\beta_{0}-\boldsymbol{\beta}^{\prime} \widetilde{\mathbf{y}}\right)^{2}\right]
$$

with respect to $\beta_{0}$ and $\boldsymbol{\beta}$. Rearranging terms

$$
M S E=\beta_{0}^{2}-2 \cdot \beta_{0} \cdot\left(E\left[\widetilde{x}_{0}\right]-\boldsymbol{\beta}^{\prime} E[\widetilde{\mathbf{y}}]\right)+E\left[\left(\widetilde{x}_{0}-\boldsymbol{\beta}^{\prime} \widetilde{\mathbf{y}}\right)^{2}\right]
$$

and taking the derivative w.r.t. $\beta_{0}$ yields

$$
\beta_{0}^{*}=E\left[\widetilde{x}_{0}\right]-\boldsymbol{\beta}^{\prime} E[\widetilde{\mathbf{y}}] .
$$

Substituting for $\beta_{0}$ from (25) into (24) yields

$$
\begin{aligned}
M S E & =E\left[\left(\widetilde{x}_{0}-\boldsymbol{\beta}^{\prime} \widetilde{\mathbf{y}}\right)^{2}\right]-\left(E\left[\widetilde{x}_{0}\right]-\boldsymbol{\beta}^{\prime} E[\widetilde{\mathbf{y}}]\right)^{2} \\
& \left.=V A R\left[\widetilde{x}_{0}-\boldsymbol{\beta}^{\prime} \widetilde{\mathbf{y}}\right)\right] \\
& =V A R\left[\widetilde{x}_{0}\right]+\boldsymbol{\beta}^{\prime} \mathbf{V} \boldsymbol{\beta}-2 \boldsymbol{\beta}^{\prime} \mathbf{d}
\end{aligned}
$$

Taking the first derivative of the last expression in (26) yields the system of first order conditions

$$
\mathbf{V} \boldsymbol{\beta}=\mathbf{d}
$$


The solution of this system is given in (11).

Part 2a: To reproduce the result in (8), note that for $n=1, \boldsymbol{\beta}=\beta_{1}, \mathbf{V}=V A R\left[\widetilde{y}_{1}\right]$ and $\mathbf{d}=\operatorname{COV}\left[\widetilde{x}_{0}, \widetilde{y}_{1}\right]$. It follows that

$$
\beta_{1}=\frac{C O V\left[\widetilde{x}_{0}, \widetilde{y}_{1}\right]}{\operatorname{VAR}\left[\widetilde{y}_{1}\right]} .
$$

Part 2b: Since (10) is a generalization of (9), it suffices to verify that (10) is a special case of (11). Let $\widetilde{x}_{0}$ and $\widetilde{y}_{i}$ have the structure given in (1) and let $c_{i}$ be a firm specific constant. It follows that

$$
\begin{aligned}
\operatorname{VAR}\left[\widetilde{y}_{i}\right] & =c_{i}^{2} \cdot \operatorname{VAR}[\widetilde{\eta}]+V A R\left[\widetilde{\varepsilon}_{i}\right] \\
\operatorname{COV}\left[\widetilde{y}_{i}, \widetilde{y}_{j}\right] & =c_{i} \cdot c_{j} \cdot \operatorname{VAR}[\widetilde{\eta}] \\
\operatorname{COV}\left[\widetilde{x}_{0}, \widetilde{y}_{i}\right] & =c_{0} \cdot c_{i} \cdot V A R[\widetilde{\eta}]
\end{aligned}
$$

With this structure, the equation system in (27) takes the form

$$
\left(\begin{array}{ccc}
c_{1}^{2} \cdot V A R[\widetilde{\eta}]+V A R\left[\widetilde{\varepsilon}_{1}\right] & \ldots & c_{1} \cdot c_{n} \cdot V A R[\widetilde{\eta}] \\
\vdots & \ddots & \vdots \\
c_{1} \cdot c_{n} \cdot V A R[\widetilde{\eta}] & \cdots & c_{n}^{2} \cdot V A R[\widetilde{\eta}]+V A R\left[\widetilde{\varepsilon}_{n}\right]
\end{array}\right)\left(\begin{array}{c}
\beta_{1} \\
\vdots \\
\beta_{n}
\end{array}\right)=\left(\begin{array}{c}
c_{0} \cdot c_{1} \cdot V A R[\widetilde{\eta}] \\
\vdots \\
c_{0} \cdot c_{n} \cdot V A R[\widetilde{\eta}]
\end{array}\right),
$$

dividing each row of this system by $c_{0} \cdot c_{i} \cdot V A R[\tilde{\eta}]$ yields the simplified system

$$
\mathbf{H} \boldsymbol{\beta}=\mathbf{1},
$$

where 1 is the $n \times 1$ ones vector,

$$
\mathbf{H}=\left(\begin{array}{ccc}
k_{1}+h_{1} & \cdots & k_{n} \\
\vdots & \ddots & \vdots \\
k_{1} & \cdots & k_{n}+h_{1}
\end{array}\right)
$$

and

$$
k_{i}=\frac{c_{i}}{c_{0}}, \quad h_{i}=\frac{V A R\left[\widetilde{\varepsilon}_{i}\right]}{c_{0} \cdot c_{i} \cdot V A R[\widetilde{\eta}]}=\frac{1}{c_{0} \cdot c_{i} \cdot \tau_{i} \cdot V A R[\widetilde{\eta}]} .
$$

Applying Cramer's rule, yields the solution

$$
\beta_{i}=\frac{\left|\mathbf{H}_{\mathbf{i}}\right|}{|\mathbf{H}|}=\frac{\prod_{j=1, j \neq i}^{n} h_{j}}{\prod_{j=1}^{n} h_{j}+\sum_{j=1}^{n} k_{j} \cdot \prod_{l=1, j \neq l}^{n} h_{l}},
$$


where $\mathbf{H}_{\mathbf{i}}$ is the matrix $\mathbf{H}$ with the $i$-th column replaced by the ones vector $\mathbf{1}$. From (31) and the fact that $\beta_{i}=\alpha \cdot \gamma_{i}$, the ratio of any two peer weights in (7) equals

$$
\frac{\gamma_{i}}{\gamma_{j}}=\frac{h_{j}}{h_{i}}=\frac{c_{i} \cdot \tau_{i}}{c_{j} \cdot \tau_{j}}
$$

Solving (32) for all $\gamma_{j}$ and substituting the resulting expression for all $j \neq i$ into the index equation

$$
\sum_{i=1}^{n} \gamma_{i}=1
$$

yields the aggregation rule in (10). Setting $c_{i}=1$ for all $i$ yields Holmstrom's aggregation rule in equation $(9)$.

\section{Proof of Corollary 1}

The optimal solution of the agency problem solves the following maximization problem:

$$
\begin{aligned}
\max U_{P} & =E\left[\widetilde{x}_{0}\right]-E\left[s\left(\widetilde{z}_{0}\right)\right] \\
\text { s.t. } U_{A} & =E\left[s\left(\widetilde{z}_{0}\right)\right]-h(r) \cdot V A R\left[s\left(\widetilde{z}_{0}\right)\right] \geq \underline{U} \\
\frac{\partial U_{A}}{\partial a_{0}} & =\frac{\partial E\left[s\left(\widetilde{z}_{0}\right)\right]}{\partial a_{0}}-C\left(a_{0}\right)=0
\end{aligned}
$$

where

$$
\begin{aligned}
E\left[\widetilde{x}_{0}\right] & =a_{0}+E\left[\widetilde{c}_{0}\right] \cdot E[\widetilde{\eta}] \\
E\left[s\left(\widetilde{x}_{0}\right)\right] & =w_{0}+v_{0} \cdot\left(E\left[\widetilde{x}_{0}\right]-\alpha \cdot E[\widetilde{y}]\right) \\
V A R\left[s\left(\widetilde{z}_{0}\right)\right] & =v_{0}^{2} \cdot V A R\left[\widetilde{z}_{0}\right]=v_{0}^{2} \cdot\left(V A R\left[\widetilde{x}_{0}\right]+\alpha^{2} V A R[\widetilde{y}]-2 \cdot \alpha \cdot \operatorname{COV}\left[\widetilde{x}_{0}, \widetilde{y}\right]\right)
\end{aligned}
$$

Let $L$ denote the Lagrangian of the principal's constrained maximization problem and let $\lambda$ be the multiplier of the participation constraint in (34). Taking the derivatives of $L$ with respect to $w_{0}$ and $\alpha$ yields:

$$
\begin{aligned}
\frac{\partial L}{\partial w_{0}} & =-1+\lambda=0 \\
\frac{\partial L}{\partial \alpha} & =-v_{0}(1-\lambda)+\lambda \cdot h(r) \cdot v_{0}^{2} \cdot \frac{\partial V A R\left[\widetilde{z}_{0}\right]}{\partial \alpha}=0 .
\end{aligned}
$$

It follows that $\alpha$ is set so that the variance of the agent's pay is minimized. This solution is independent of the optimal incentive rate $v_{0}$. An analogous result can be found if the performance measure explicitly aggregates multiple peers into a performance index and takes 
the form $\widetilde{z}_{0}=\widetilde{x}_{0}-\boldsymbol{\beta}^{\prime} \tilde{\mathbf{y}}$. Taking into account that $\lambda=1$, the optimal aggregation rule for this case must satisfy

$$
\frac{\partial L}{\partial \boldsymbol{\beta}}=\frac{\partial V A R\left[\widetilde{z}_{0}\right]}{\partial \boldsymbol{\beta}}=0 .
$$

The condition in (38) is equivalent to the optimality condition for the BLP in (27) and solved by (11) because essentially, the MSE criterion minimizes $\left.V A R\left[\widetilde{z}_{0}\right]=V A R\left[\widetilde{x}_{0}-\boldsymbol{\beta}^{\prime} \tilde{\mathbf{y}}\right)\right]$.

\section{Proof of Proposition 3}

As shown in the proof of proposition 1, the BLP for a single performance signal is given by (8). Since $\widetilde{c}_{0}, \widetilde{c}_{j}$, and $\widetilde{\eta}$ are mutually independent, the covariance between $\widetilde{x}$ and $\widetilde{y}$ equals:

$$
C O V[\widetilde{x}, \widetilde{y}]=E\left[\widetilde{c}_{0}\right] \cdot E\left[\widetilde{c}_{j}\right] \cdot V A R[\widetilde{\eta}]
$$

From (3), the variance of $\widetilde{y}$ equals

$$
V A R[\widetilde{y}]=V A R\left(\widetilde{\varepsilon}_{j}\right)+E\left[\widetilde{c}_{j}\right]^{2} \cdot V A R[\widetilde{\eta}]+R_{j}
$$

where $R_{j}$ is the exposure risk as defined in (4)

$$
R_{j}=V A R\left[\widetilde{c}_{j}\right] \cdot\left(V A R[\widetilde{\eta}]+E[\widetilde{\eta}]^{2}\right)
$$

With this structure, the optimal aggregation rule becomes

$$
\alpha^{\circ}=\frac{E\left[\widetilde{c}_{0}\right] \cdot E\left[\widetilde{c}_{j}\right] \cdot V A R[\widetilde{\eta}]}{V A R\left(\widetilde{\varepsilon}_{j}\right)+E\left[\widetilde{c}_{j}\right]^{2} \cdot V A R[\widetilde{\eta}]+R_{j}} .
$$

It holds that

$$
\begin{aligned}
\frac{\partial \alpha^{\circ}}{\partial R_{j}} & =\frac{\partial \alpha^{\circ}}{\partial V A R\left(\widetilde{\varepsilon}_{j}\right)}=-\frac{C O V[\widetilde{x}, \widetilde{y}]}{V A R[\widetilde{y}]^{2}}<0 \\
\frac{\partial \alpha^{\circ}}{\partial V A R\left[\widetilde{c}_{j}\right]} & =\left(V A R[\widetilde{\eta}]+E[\widetilde{\eta}]^{2}\right) \cdot \frac{\partial \alpha^{\circ}}{\partial R_{j}}<0 \\
\frac{\partial \alpha^{\circ}}{\partial V A R[\widetilde{\eta}]} & =\frac{E\left[\widetilde{c}_{0}\right] \cdot E\left[\widetilde{c}_{j}\right] \cdot\left(V A R\left(\widetilde{\varepsilon}_{j}\right)+V A R\left[\widetilde{c}_{j}\right] \cdot E[\widetilde{\eta}]^{2}\right)}{V A R[\widetilde{y}]^{2}}>0 \\
\frac{\partial \alpha^{\circ}}{\partial E\left[\widetilde{c}_{0}\right]} & =\frac{E\left[\widetilde{c}_{j}\right] \cdot V A R[\widetilde{\eta}]}{V A R[\widetilde{y}]}>0 \\
\frac{\partial \alpha^{\circ}}{\partial E\left[\widetilde{c}_{j}\right]} & =\frac{E\left[\widetilde{c}_{0}\right] \cdot V A R[\widetilde{\eta}]}{V A R[\widetilde{y}]^{2}} \cdot \Delta_{j}
\end{aligned}
$$


where $\Delta_{j}$ in (44) is given by the expression

$$
\Delta_{j}=V_{j}-E\left[\widetilde{c}_{j}\right]^{2} \cdot V A R[\tilde{\eta}] .
$$

The expression in (45) can be positive or negative. It is strictly positive if $V_{j}>E\left[\widetilde{c}_{j}\right]^{2} \cdot V A R[\widetilde{\eta}]$ where $V_{j}=\operatorname{VAR}\left(\widetilde{\varepsilon}_{j}\right)+R_{j}$ is the sum of the idiosyncratic risk $V A R\left(\widetilde{\varepsilon}_{j}\right)$ and the exposure risk $R_{j}$ contained in the performance measure $\widetilde{y}$ as defined in (4).

\section{Proof of Proposition 4}

The proof of proposition 4 is similar to the second part of the proof of proposition 1 . In the presence of exposure risk, the elements of the covariance matrix $\mathbf{V}$ and the covariance vector $\mathbf{d}$ in (27) become:

$$
\begin{aligned}
V A R\left[\widetilde{y}_{i}\right] & =V A R\left(\widetilde{\varepsilon}_{i}\right)+E\left[\widetilde{c}_{i}\right]^{2} \cdot V A R[\widetilde{\eta}]+R_{i} \\
\operatorname{COV}\left[\widetilde{y}_{i}, \widetilde{y}_{j}\right] & =E\left[\widetilde{c}_{i}\right] \cdot E\left[\widetilde{c}_{j}\right] \cdot V A R[\widetilde{\eta}] \\
\operatorname{COV}\left[\widetilde{x}_{0}, \widetilde{y}_{i}\right] & =E\left[\widetilde{c}_{0}\right] \cdot E\left[\widetilde{c}_{i}\right] \cdot V A R[\widetilde{\eta}]
\end{aligned}
$$

where $R_{i}=V A R\left[\widetilde{c}_{i}\right] \cdot\left(V A R[\widetilde{\eta}]+E[\widetilde{\eta}]^{2}\right)$ is the exposure risk embedded in $\widetilde{y}_{i}$. With these definitions, the matrix $\mathbf{H}$ in (29) that determines the solution of the modified system in (28) has entries

$$
k_{i}=\frac{E\left[c_{i}\right]}{E\left[c_{0}\right]}, h_{i}=\frac{V A R\left[\widetilde{\varepsilon}_{i}\right]+R_{i}}{E\left[\widetilde{c}_{0}\right] \cdot E\left[\widetilde{c}_{i}\right] \cdot V A R[\widetilde{\eta}]} .
$$

Now, let

$$
\delta_{i}\left(R_{i}\right)=\frac{1}{V_{i}}=\frac{1}{V A R\left[\widetilde{\varepsilon}_{i}\right]+R_{i}}
$$

where $V_{j}$ is the sum of the idiosyncratic risk $V A R\left[\widetilde{\varepsilon}_{j}\right]$ and the exposure risk $R_{i}$ embedded in $\widetilde{y}_{i}$ as defined in equation (4). It follows that

$$
\frac{\beta_{i}}{\beta_{j}}=\frac{\gamma_{i}}{\gamma_{j}}=\frac{h_{j}}{h_{i}}=\frac{E\left[c_{i}\right] \cdot \delta_{i}\left(R_{i}\right)}{E\left[c_{j}\right] \cdot \delta_{j}\left(R_{j}\right)} .
$$

Solving (48) for all $\gamma_{j}$ and substituting the resulting expression for all $j \neq i$ into the index equation $\sum_{i=1}^{n} \gamma_{i}=1$, yields the aggregation rule

$$
\gamma_{j}^{+}=\frac{E\left[\widetilde{c}_{j}\right] \cdot \delta_{j}\left(R_{j}\right)}{\sum_{j=1}^{n} E\left[\widetilde{c}_{j}\right] \cdot \delta_{j}\left(R_{j}\right)}
$$


1) It holds that

$$
\begin{aligned}
\frac{\partial \gamma_{j}^{+}}{\partial \delta_{j}} & =\frac{E\left[\widetilde{c}_{j}\right] \cdot \sum_{k=1, k \neq j}^{n} E\left[\widetilde{c}_{k}\right] \cdot \delta_{k}}{\left(\sum_{j=1}^{n} E\left[\widetilde{c}_{j}\right] \cdot \delta_{j}\right)^{2}}>0 \\
\frac{\partial \gamma_{j}^{+}}{\partial E\left[\widetilde{c}_{j}\right]} & =\frac{\delta_{j} \cdot \sum_{k=1, k \neq j}^{n} E\left[\widetilde{c}_{k}\right] \cdot \delta_{k}}{\left(\sum_{j=1}^{n} E\left[\widetilde{c}_{j}\right] \cdot \delta_{j}\right)^{2}}>0
\end{aligned}
$$

and, for any peer with $k \neq j$

$$
\begin{aligned}
\frac{\partial \gamma_{j}^{+}}{\partial \delta_{k}} & =-\frac{\delta_{j} \cdot E\left[\widetilde{c}_{j}\right] \cdot E\left[\widetilde{c}_{k}\right]}{\left(\sum_{j=1}^{n} E\left[\widetilde{c}_{j}\right] \cdot \delta_{j}\right)^{2}}<0 \\
\frac{\partial \gamma_{j}^{+}}{\partial E\left[\widetilde{c}_{k}\right]} & =-\frac{\delta_{j} \cdot E\left[\widetilde{c}_{j}\right] \cdot \delta_{k}}{\left(\sum_{j=1}^{n} E\left[\widetilde{c}_{j}\right] \cdot \delta_{j}\right)^{2}}<0
\end{aligned}
$$

2) Since $\delta_{j}\left(R_{j}\right)=\frac{1}{V A R\left[\widetilde{\varepsilon}_{j}\right]+R_{j}}$, it holds that

$$
\frac{\partial \delta_{j}\left(R_{j}\right)}{\partial R_{j}}=-\frac{1}{\left(V A R\left[\widetilde{\varepsilon}_{j}\right]+R_{j}\right)^{2}} \quad \text { and } \quad \frac{\partial \gamma_{j}^{+}}{\partial R_{j}}=\frac{\partial \gamma_{j}^{+}}{\partial \delta_{j}} \frac{\partial \delta_{j}\left(R_{j}\right)}{\partial R_{j}}<0 .
$$

Moreover,

$$
\lim _{R_{j} \rightarrow \infty} \gamma_{j}^{+}=\lim _{R_{j} \rightarrow \infty} \frac{E\left[\widetilde{c}_{j}\right]}{E\left[\widetilde{c}_{j}\right]+\left(V A R\left[\widetilde{\varepsilon}_{j}\right]+R_{j}\right) \cdot \sum_{k=1, k \neq j}^{n} E\left[\widetilde{c}_{k}\right] \cdot \delta_{k}}=0 .
$$

\section{Proof of Corollary 5}

Plugging the definitions from (46) into (31) yields

$$
\beta_{j}^{+}=\frac{E\left[\widetilde{c}_{0}\right] \cdot V A R[\widetilde{\eta}] \cdot E\left[\widetilde{c}_{j}\right] \cdot \delta_{j}\left(R_{j}\right)}{1+V A R[\widetilde{\eta}] \cdot \sum_{j=1}^{n} E\left[c_{j}\right]^{2} \cdot \delta_{j}\left(R_{j}\right)} .
$$

Using the result in (49), the optimal index weight becomes

$$
\alpha^{+}=\frac{\beta_{j}^{+}}{\gamma_{j}^{+}}=\frac{E\left[\widetilde{c}_{0}\right] \cdot V A R[\widetilde{\eta}] \cdot \sum_{j=1}^{n} E\left[\widetilde{c}_{j}\right] \cdot \delta_{j}\left(R_{j}\right)}{1+V A R[\widetilde{\eta}] \cdot \sum_{j=1}^{n} E\left[c_{j}\right]^{2} \cdot \delta_{j}\left(R_{j}\right)} .
$$

Substituting the expressions for $\gamma_{j}^{+}$and $\alpha^{+}$into the $V A R\left[\widetilde{z}_{0}\right]$ yields (21). The expression in (21) is increasing in $z^{+}$because $\partial q^{+} / \partial z^{+}>0$.

The limits of the scaling factor are determined as follows:

1) Since $z^{+}=V A R[\widetilde{\eta}] \cdot \sum_{j=1}^{n} E\left[\widetilde{c}_{j}\right]^{2} \cdot \delta_{j}\left(R_{j}\right)>0$ it must be that $q^{+}<1$. 
2) To show that $q^{+}>q^{\circ}$, recall first from (18) that

$$
q^{\circ}=\frac{V A R[\widetilde{\eta}] \cdot E\left[\widetilde{c}_{j}\right]^{2}}{V A R[\widetilde{y}]}=\frac{V A R[\widetilde{\eta}] \cdot E\left[\widetilde{c}_{j}\right]^{2}}{V A R\left[\widetilde{\varepsilon}_{j}\right]+V A R[\widetilde{\eta}] \cdot E\left[c_{j}\right]^{2}+R_{j}},
$$

using the definition of $\delta_{j}\left(R_{j}\right)$ and rearranging terms yields

$$
q^{\circ}=\frac{z^{\circ}}{1+z^{\circ}}
$$

where $z^{\circ}=V A R[\widetilde{\eta}] \cdot E\left[\widetilde{c}_{j}\right]^{2} \cdot \delta_{j}\left(R_{j}\right)$. If firm $j$ is part of the index, it must be that

$$
E\left[\widetilde{c}_{j}\right]^{2} \cdot \delta_{j}\left(R_{j}\right)<\sum_{j=1}^{n} E\left[\widetilde{c}_{j}\right]^{2} \cdot \delta_{j}\left(R_{j}\right)
$$

It follows that $z^{+}>z^{\circ}$ and that $q^{+}>q^{\circ}$.

3) Using the expressions in (14), (18), and (21), the range of $q^{+}$implies that

$$
V A R\left[\widetilde{z}_{0}\left(\alpha^{\circ}\right)\right]>V A R\left[\widetilde{z}_{0}\left(\alpha^{+}\right)\right]>V A R\left[\widetilde{z}_{0}\left(\alpha^{*}\right)\right]
$$

\section{References}

Aabo, T. (2015): Corporate Hedging of Price Risks: Minimizing Variance or Eliminating Lower-Tail Outcomes?. Journal of Applied Corporate Finance 27(1), 57-62.

Aggarwal, R. and A. Samwick, A. (1999): Executive compensation, strategic competition, and relative performance evaluation: Theory and evidence. Journal of Finance 54(6), 1999-2043.

Albuquerque, A. (2009): Peer firms in relative performance evaluation. Journal of Accounting and Economics 48 (1), 69-89.

Albuquerque, A. (2014): Do Growth-Option Firms Use Less Relative Performance Evaluation? The Accounting Review: 89(1), 27-60.

Allayannis, G. and J. Ihrig (2001): Exposure and Markups. Review of Financial Studies $14(3), 805-835$. 
Amemiya, T. (1994): Introduction to Statistics and Econometrics. Harvard University Press, Cambridge and London.

Ang, A. and Chen, J. (2007): CAPM over the long run: 1926-2001. Journal of Empirical Finance 14, 1-40.

Aroian, L.A. (1947): The Probability Function of the Product of Two Normally Distributed Variables. The Annals of Mathematical Statistics 18 (2), 265-271.

Banker, R. D. and S. M. Datar (1989): Sensitivity, precision, and linear aggregation of signals for performance evaluation. Journal of Accounting Research 27(1), 21-39.

Bannister,J. and H. Newman (2003): An analysis of corporate disclosures on relative performance evaluation. Accounting Horizons 17 (3), 235-246.

Bertrand, M. and S. Mullainathan (2001): Are CEOs rewarded for Luck? The Ones without Principals are. The Quarterly Journal of Economics 116(3), 901-932.

Bohrnstedt, G. W. and A. S. Goldberger (1969): On the Exact Covariance of Products of Random Variables. Journal of the American Statistical Association 64(328), 14391442.

Craig (1936): On the Frequency Function of xy. The Annals of Mathematical Statistics 7(1), 1-15.

Engle, R. (2009): Anticipating Correlations, A New Paradigm of risk Management, Princeton University Press, Princeton and Oxford.

Epstein, B. (1948): Some Applications of the Mellin Transform in Statistics. The Annals of Mathematical Statistics 19 (3), 370-379.

Dikolli, S., Hofmann, C. and T. Pfeiffer (2013): Relative performance evaluation and peerperformance summarization errors. Review of Accounting Studies 18 (1), 34-65.

Dikolli, S., Diser, V. Hofmann, C. and T. Pfeiffer (2014): CEO Power and relative performance evaluation. Working paper, presented at the AAA MAS midyear meeting 2014, available from the AAA website: aaahq.org/MAS/meetings/2014/program.cfm. 
Fama, E. and French, K. (1997): Industry cost of equity. Journal of Financial Economics 43, 153-93.

Ferriozi, F. (2011): Paying for observable luck. Rand Journal of Economics 42(2), 387-415.

Francis, B., Hasan, I. and D. Hunter (2008): Can hedging tell the full story? Reconciling differences in United States aggregate- and industry-level exchange rate risk premium. Journal of Financial Economics 90 (2), 169-196.

Garvey, G. and T. Milbourn (2003): Incentive Compensation When Executives Can Hedge the Market: Evidence of Relative Performance Evaluation in the Cross Section. Journal of Finance 58 (4), 1557-1581.

Garvey, G. and T. Milbourn (2006): Asymmetric benchmarking in compensation: Executives are rewarded for good luck but not penalized for bad. Journal of Financial Economics 82 (1), 197-225.

Fershtman, C. Hvide H. and Y. Weiss. (2003): A Behavioral Explanation of the Relative Performance Evaluation Puzzle. Annals D'économie et de Statistique (71/72), 349-361.

Göx, R. (2008): Tax Incentives for Inefficient Executive Pay and Reward for Luck. Review of Accounting Studies 13, 452-478.

Goldberger, A. S. (1991): A Course in Econometrics. Harvard University Press, Cambridge and London.

Goodman. L. A. (1960): On the Exact Variance of Products. Journal of the American Statistical Association 55, 708-713.

Gong, G. Li, L. and J. Y. Shin (2011): Relative Performance Evaluation and Related Peer Groups in Executive Compensation Contracts. The Accounting Review 86(3), 1007-1043.

Gopalan, R., Milbourn, T. and F. Song (2010): Strategic Flexibility and the Optimality of Pay for Sector Performance. Review of Financial Studies 23(5), 2060-2098.

Greene, W. H. (2008): Econometric Analysis, 6th edition. Pearson Prentice Hall, Upper Saddle River. 
Hermalin, B. E. and N. E. Wallace (2001): Firm performance and executive compensation in the savings and loan industry. Journal of Financial Economics 61(1), 139-170.

Holmstrom, B. (1979): Moral Hazard and Observability, The Bell Journal of Economics 10(1), 74-91.

Holmstrom, B. (1982): Moral hazard in teams. The Bell Journal of Economics 13(2), $324-340$.

Holmstrom, B. and P. Milgrom (1987): Aggregation and linearity in the provision of intertemporal incentives. Econometrica 55(2), 303-328.

Hayya J.C. and W.L. Ferrara (1972): On Normal Approximations of the Frequency Functions of Standard Forms Where the Main Variables are Normally Distributed. Management Science 19(2), 173-186.

Jagannathan, R. and Wang, Z. (1996): The conditional CAPM and the cross-section of expected returns. Journal of Finance 51, 3-53.

Kerkvliet, J. and M. H. Moffett (1991): The Hedging of an Uncertain Future Foreign Currency Cash Flow. Journal of Financial and Quantitative Analysis 26(4), 565-578.

Lewellen, J. and Nagel, S. (2006): The conditional CAPM does not explain asset-pricing anomalies. Journal of Financial Economics 82, 289-314.

Maug, E. (2000): The relative performance puzzle. Schmalenbach Business Review 52, $3-24$. 
Table 1

PANEL A shows the relevant parameters used for simulating firm performance $\tilde{x}_{0}=k_{0}+\tilde{c}_{0} \cdot \tilde{\eta}+\tilde{\varepsilon}_{0}$ and common risk $\tilde{\eta}$. For each regression 1,000 observations were randomly generated for each variable using the parameter $k_{0}=1$ and independent random numbers generated from the distributions: $\tilde{\eta} \sim \mathrm{N}(1,1), \tilde{c}_{0} \sim \mathrm{N}\left(1, \sigma_{0}^{2}\right), \tilde{\varepsilon}_{0} \sim \mathrm{N}(0,1)$.

PANEL B presents the summary statistics of 1,000 estimations of the regression model $x_{0 i}=a_{0}+a_{1} \cdot \eta_{i}+\epsilon_{i}$ using simulated data for the 6 parameter sets and random numbers as defined in PANEL A.

PANEL C presents the summary statistics of 1,000 estimations of the regression model $z_{0 i}=b_{0}+b_{1} \cdot \eta_{i}+e_{i}$ where $z_{0 i}=x_{0 i}-E\left[\tilde{c}_{0}\right] \cdot \eta_{i}$ and $E\left[\tilde{c}_{0}\right]=1$. All regressions are estimates using the same data as for the regressions displayed in PANEL B.

PANEL A: Parameters for the standard deviation of $\tilde{c}_{0}$ used for simulating firm performance

\begin{tabular}{|c|c|c|c|c|c|c|}
\hline & \multicolumn{6}{|c|}{ Regression model } \\
\hline & $(1)$ & $(2)$ & (3) & $(4)$ & $(5)$ & $(6)$ \\
\hline$\sigma_{0}$ & 0 & 1 & 3 & 6 & 9 & 12 \\
\hline \multicolumn{7}{|c|}{ PANEL B: Simulated regressions of unfiltered firm performance on common risk } \\
\hline & $(1)$ & $(2)$ & (3) & (4) & (5) & (6) \\
\hline \multicolumn{7}{|l|}{ Risk exposure $\left(\hat{a}_{1}\right)$} \\
\hline Mean & 1.0002 & 0.9976 & 1.0015 & 0.9939 & 0.9978 & 0.9964 \\
\hline Standard deviation & 0.0320 & 0.0723 & 0.1935 & 0.3661 & 0.5756 & 0.7686 \\
\hline Min & 0.8914 & 0.7770 & 0.2502 & -0.1868 & -1.2238 & -1.2425 \\
\hline Max & 1.0888 & 1.2850 & 1.5708 & 2.0242 & 2.6055 & 3.9774 \\
\hline \multicolumn{7}{|l|}{ t-values } \\
\hline Mean & 31.6062 & 18.1978 & 7.2833 & 3.6846 & 2.4794 & 1.8576 \\
\hline Standard deviation & 1.4744 & 1.4583 & 1.4370 & 1.3667 & 1.4321 & 1.4405 \\
\hline $\begin{array}{l}\text { Share of } \hat{a}_{1} \text {-coefficients } \\
\text { significant at } 95 \% \text {-level }\end{array}$ & $100 \%$ & $100 \%$ & $99.9 \%$ & $88.6 \%$ & $62.8 \%$ & $46.6 \%$ \\
\hline \multicolumn{7}{|l|}{ R-squared } \\
\hline Mean & 0.4997 & 0.2492 & 0.0520 & 0.0151 & 0.0081 & 0.0055 \\
\hline Standard deviation & 0.0234 & 0.0298 & 0.0188 & 0.0100 & 0.0073 & 0.0061 \\
\hline Observations & 1,000 & 1,000 & 1,000 & 1,000 & 1,000 & 1,000 \\
\hline
\end{tabular}

PANEL C: Simulated regressions of filtered firm performance on common risk

\begin{tabular}{lrrrrrr}
\hline & $(1)$ & $(2)$ & $(3)$ & $(4)$ & $(5)$ & $(6)$ \\
\hline Risk exposure $\left(\hat{b}_{1}\right)$ & & & & & & \\
$\quad$ Mean & 0.0002 & -0.0024 & 0.0015 & -0.0061 & -0.0022 & -0.0036 \\
Standard deviation & 0.0320 & 0.0723 & 0.1935 & 0.3661 & 0.5756 & 0.7686 \\
Min & -0.1086 & -0.2230 & -0.7498 & -1.1868 & -2.2238 & -2.2425 \\
Max & 0.0888 & 0.2850 & 0.5708 & 1.0242 & 1.6055 & 2.9774 \\
t-values & & & & & & \\
$\quad$ Mean & 0.0080 & -0.0435 & 0.0116 & -0.0204 & -0.0052 & -0.0039 \\
$\quad$ Standard deviation & 1.0097 & 1.3180 & 1.4055 & 1.3526 & 1.4283 & 1.4350 \\
Share of $\hat{b}_{1}$-coefficients & & & & & & \\
significant at 95\%-level & $5.7 \%$ & $14.3 \%$ & $17.5 \%$ & $14.9 \%$ & $17.0 \%$ & $18.2 \%$ \\
R-squared & & & & & & \\
$\quad$ Mean & 0.0010 & 0.0017 & 0.0020 & 0.0018 & 0.0020 & 0.0020 \\
$\quad$ Standard deviation & 0.0014 & 0.0025 & 0.0027 & 0.0025 & 0.0027 & 0.0029 \\
$\quad$ Observations & 1,000 & 1,000 & 1,000 & 1,000 & 1,000 & 1,000 \\
\hline
\end{tabular}


Table 2

PANEL A shows the relevant parameters used for simulating firm performance $\tilde{x}_{0}=k_{0}+\tilde{c}_{0} \cdot \tilde{\eta}+\tilde{\varepsilon}_{0}$ and a noisy measure of common risk $\tilde{y}=k_{1}+\tilde{c}_{1} \cdot \tilde{\eta}+\tilde{\varepsilon}_{1}$. For each regression 1,000 observations were randomly generated for each variable using independent random numbers generated from the distributions $\tilde{\eta} \sim \mathrm{N}(1,1), \tilde{\varepsilon}_{0}, \tilde{\varepsilon}_{1} \sim \mathrm{N}(0,1)$, $\tilde{c}_{0} \sim \mathrm{N}(1,1), \tilde{c}_{1} \sim \mathrm{N}\left(1, \sigma_{1}^{2}\right)$ with parameters $k_{0}=k_{1}=1$, and the values for $\sigma_{1}$ given below.

PANEL B presents the summary statistics of 1,000 estimations of the regression model $x_{0 i}=a_{0}+a_{1} \cdot y_{i}+\epsilon_{i}$ using simulated data for the 6 parameter sets and random numbers as defined in PANEL A.

PANEL C presents the summary statistics of 1,000 estimations of the regression model $z_{0 i}=b_{0}+b_{1} \cdot y_{i}+e_{i}$ where $z_{0 i}=x_{0 i}-\alpha^{\circ} \cdot y_{i}$ where $\alpha^{\circ}$ is the optimal weight of $\tilde{y}$ as defined in equation (16).

PANEL A: Parameters for the standard deviation of $\tilde{c}_{1}$ used for simulating the noisy signal of common risk

Regression model

\begin{tabular}{lcccccc}
\cline { 2 - 6 } & $(1)$ & $(2)$ & $(3)$ & $(4)$ & $(5)$ & $(6)$ \\
\hline$\sigma_{1}$ & 0 & 1 & 3 & 6 & 12 \\
\hline
\end{tabular}

PANEL B: Simulated regressions of unfiltered firm performance on noisy signal of common risk

\begin{tabular}{|c|c|c|c|c|c|c|}
\hline & $(1)$ & $(2)$ & (3) & (4) & (5) & $(6)$ \\
\hline \multicolumn{7}{|l|}{ Risk exposure $\left(\hat{a}_{1}\right)$} \\
\hline Mean & 0.5032 & 0.2500 & 0.0507 & 0.0142 & 0.0060 & 0.0036 \\
\hline Standard deviation & 0.0465 & 0.0373 & 0.0205 & 0.0102 & 0.0069 & 0.0052 \\
\hline Min & 0.3613 & 0.1226 & -0.0190 & -0.0230 & -0.0153 & -0.0127 \\
\hline Max & 0.6494 & 0.3779 & 0.1179 & 0.0449 & 0.0298 & 0.0181 \\
\hline \multicolumn{7}{|l|}{ t-values } \\
\hline Mean & 12.0299 & 8.1555 & 3.6105 & 1.9425 & 1.2143 & 0.9715 \\
\hline Standard deviation & 1.1301 & 1.2623 & 1.4693 & 1.3878 & 1.4016 & 1.3928 \\
\hline $\begin{array}{l}\text { Share of } \hat{a}_{1} \text {-coefficients } \\
\text { significant at } 95 \% \text {-level }\end{array}$ & $100.0 \%$ & $100.0 \%$ & $87.2 \%$ & $49.8 \%$ & $30.8 \%$ & $26.2 \%$ \\
\hline \multicolumn{7}{|l|}{ R-squared } \\
\hline Mean & 0.1271 & 0.0635 & 0.0149 & 0.0056 & 0.0034 & 0.0029 \\
\hline Standard deviation & 0.0207 & 0.0182 & 0.0106 & 0.0059 & 0.0042 & 0.0035 \\
\hline Observations & 1,000 & 1,000 & 1,000 & 1,000 & 1,000 & 1,000 \\
\hline
\end{tabular}

PANEL C: Simulated regressions of filtered firm performance on noisy signal of common risk

\begin{tabular}{|c|c|c|c|c|c|c|}
\hline & $(1)$ & $(2)$ & (3) & (4) & (5) & (6) \\
\hline \multicolumn{7}{|l|}{ Risk exposure $\left(\hat{b}_{1}\right)$} \\
\hline Mean & 0.0032 & 0.0000 & 0.0007 & 0.0007 & -0.0001 & 0.0002 \\
\hline Standard deviation & 0.0465 & 0.0373 & 0.0205 & 0.0102 & 0.0069 & 0.0052 \\
\hline Min & -0.1387 & -0.1274 & -0.0690 & -0.0365 & -0.0214 & -0.0161 \\
\hline Max & 0.1494 & 0.1279 & 0.0679 & 0.0314 & 0.0237 & 0.0147 \\
\hline \multicolumn{7}{|l|}{ t-values } \\
\hline Mean & 0.0711 & 0.0013 & 0.0453 & 0.0974 & -0.0210 & 0.0410 \\
\hline Standard deviation & 1.1116 & 1.2188 & 1.4624 & 1.3869 & 1.3986 & 1.3935 \\
\hline $\begin{array}{l}\text { Share of } \hat{b}_{1} \text {-coefficients } \\
\text { significant at } 95 \% \text {-level }\end{array}$ & $8.3 \%$ & $10.3 \%$ & $18.1 \%$ & $15.8 \%$ & $15.7 \%$ & $16.6 \%$ \\
\hline \multicolumn{7}{|l|}{ R-squared } \\
\hline Mean & 0.0012 & 0.0015 & 0.0021 & 0.0019 & 0.0019 & 0.0019 \\
\hline Standard deviation & 0.0018 & 0.0021 & 0.0030 & 0.0028 & 0.0027 & 0.0026 \\
\hline Observations & 1,000 & 1,000 & 1,000 & 1,000 & 1,000 & 1,000 \\
\hline
\end{tabular}




\section{Table 3}

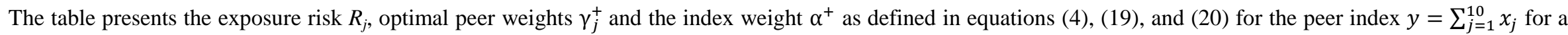

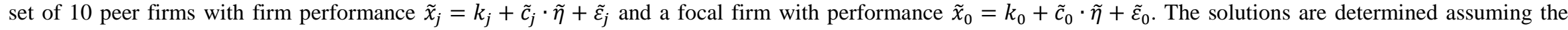

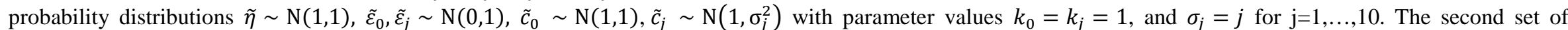
entries compares the results to the same set of peers in the absence of exposure risk, i.e. $\sigma_{j}=0$ for $\mathrm{j}=1, \ldots, 10$.

Table 3: Aggregation of peer groups with and without exposure risk

\begin{tabular}{|c|c|c|c|c|c|c|c|c|c|c|}
\hline & \multicolumn{10}{|c|}{ Peer firm $/ \sigma_{j}$} \\
\hline & 1 & 2 & 3 & 4 & 5 & 6 & 7 & 8 & 9 & 10 \\
\hline \multicolumn{11}{|l|}{ RPE with exposure risk } \\
\hline Exposure risk $\left(R_{j}\right)$ & 2 & 8 & 18 & 32 & 50 & 72 & 98 & 128 & 162 & 200 \\
\hline Peer weight $\left(\gamma_{j}^{+}\right)$ & 0.5653 & 0.1884 & 0.0893 & 0.0514 & 0.0333 & 0.0232 & 0.0171 & 0.0131 & 0.0104 & 0.0084 \\
\hline Effective weight $\left(\gamma_{j}^{+} \cdot \alpha^{+}\right)$ & 0.2097 & 0.0699 & 0.0331 & 0.0191 & 0.0123 & 0.0086 & 0.0064 & 0.0049 & 0.0039 & 0.0031 \\
\hline \multicolumn{11}{|l|}{ RPE absent exposure risk } \\
\hline Peer weight $\left(\gamma_{j}^{n}\right)$ & 0.1 & 0.1 & 0.1 & 0.1 & 0.1 & 0.1 & 0.1 & 0.1 & 0.1 & 0.1 \\
\hline Index weight $\left(\alpha^{n}\right)$ & 0.7273 & & & & & & & & & \\
\hline Effective weight $\left(\gamma_{j}^{n} \cdot \alpha^{n}\right)$ & 0.0727 & 0.0727 & 0.0727 & 0.0727 & 0.0727 & 0.0727 & 0.0727 & 0.0727 & 0.0727 & 0.0727 \\
\hline \multicolumn{11}{|l|}{ \% Differences } \\
\hline $\begin{array}{l}\left(\gamma_{j}^{+}-\gamma_{j}^{n}\right) / \gamma_{j}^{n} \\
\left(\alpha^{+}-\alpha^{n}\right) / \alpha^{n}\end{array}$ & $\begin{array}{l}465.31 \% \\
-49.00 \%\end{array}$ & $88.44 \%$ & $-10.74 \%$ & $-48.61 \%$ & $-66.75 \%$ & $-76.77 \%$ & $-82.87 \%$ & $-86.85 \%$ & $-89.60 \%$ & $-91.56 \%$ \\
\hline
\end{tabular}




\section{Table 4}

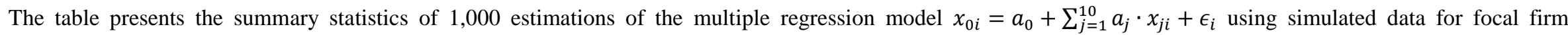

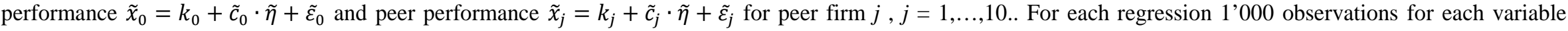

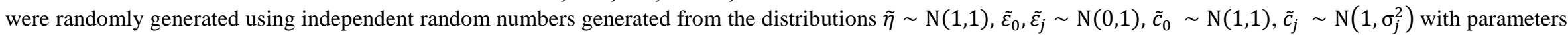
$k_{0}=k_{j}=1$, and $\sigma_{j}=j$ for $j=1, \ldots, 10$.

Table 4: Simulated regression of focal firm performance on peer performance

\begin{tabular}{|c|c|c|c|c|c|c|c|c|c|c|}
\hline & \multicolumn{10}{|c|}{ Peer firm $/ \sigma_{j}$} \\
\hline & 1 & 2 & 3 & 4 & 5 & 6 & 7 & 8 & 9 & 10 \\
\hline \multicolumn{11}{|l|}{$\begin{array}{l}\text { Firm to peer performance } \\
\text { exposure }\left(\hat{a}_{j}\right)\end{array}$} \\
\hline Mean & 0.2069 & 0.0716 & 0.0327 & 0.0196 & 0.0127 & 0.0089 & 0.0066 & 0.0046 & 0.0038 & 0.0028 \\
\hline Standard deviation & 0.0406 & 0.0276 & 0.0193 & 0.0147 & 0.0115 & 0.0096 & 0.0085 & 0.0073 & 0.0067 & 0.0060 \\
\hline Min & 0.0742 & -0.0231 & -0.0209 & -0.0238 & -0.0253 & -0.0252 & -0.0249 & -0.0201 & -0.0162 & -0.0200 \\
\hline Max & 0.3297 & 0.1559 & 0.1025 & 0.0730 & 0.0458 & 0.0472 & 0.0350 & 0.0283 & 0.0271 & 0.0221 \\
\hline \multicolumn{11}{|l|}{ t-values } \\
\hline Mean & 6.6597 & 3.6747 & 2.3908 & 1.8763 & 1.5031 & 1.2638 & 1.0772 & 0.8587 & 0.8084 & 0.6626 \\
\hline Standard deviation & 1.3257 & 1.4228 & 1.4086 & 1.4070 & 1.3587 & 1.3664 & 1.3945 & 1.3744 & 1.3998 & 1.3935 \\
\hline $\begin{array}{l}\text { Share of } \hat{a}_{j} \text {-coefficients } \\
\text { significant at } 95 \% \text {-level }\end{array}$ & $100.0 \%$ & $88.0 \%$ & $62.2 \%$ & $47.7 \%$ & $37.0 \%$ & $31.8 \%$ & $28.4 \%$ & $22.1 \%$ & $22.7 \%$ & $20.4 \%$ \\
\hline \multicolumn{11}{|l|}{ Adjusted R-squared } \\
\hline Standard deviation & 0.0236 & & & & & & & & & \\
\hline Observations & 1,000 & & & & & & & & & \\
\hline
\end{tabular}


Table 5: Simulation of pooled regression for a firm sample

PANEL A shows the relevant parameters used for simulating firm performance $\tilde{x}_{i t}=k+\tilde{c}_{i t} \cdot \tilde{\eta}_{t}+\tilde{\varepsilon}_{i t}$ and exposure to common risk $\tilde{\eta}_{t}$. For each regression a sample of 100 firms with 1,000 observations per firm was randomly generated using the parameter $k=1$ and independent random numbers generated from the distributions $\tilde{\eta}_{t} \sim$ $\mathrm{N}(1,1), \tilde{c}_{i t} \sim \mathrm{N}\left(1, \sigma_{i}^{2}\right)$, and $\tilde{\varepsilon}_{i t} \sim \mathrm{N}(0,1)$. The firm specific standard deviations of the exposure to common risk, $\sigma_{i}$, were drawn once from the uniform distributions given in the first row of PANEL A at the beginning of the simulation study.

PANEL B presents the summary statistics of 1,000 estimations of the regression model $z_{i t}=b_{0}+b_{1} \cdot \eta_{t}+e_{i t}$ where $z_{i t}=x_{i t}-E\left[\tilde{c}_{i t}\right] \cdot \eta_{t}$ and $E\left[\tilde{c}_{i t}\right]=1$.

PANEL A: Parameter distribution for the standard deviation of $\tilde{c}_{i t}$ used for simulating firm performance

\begin{tabular}{lrrrr}
\hline & & Regression model & & $(4)$ \\
\cline { 2 - 4 } & $(1)$ & $(2)$ & $(3)$ & $\mathrm{U}[0,3]$ \\
Distribution of $\sigma_{i}$ & - & $\mathrm{U}[0,1]$ & $\mathrm{U}[0,2]$ & 1.5021 \\
Sample Mean & 0 & 0.5007 & 1.0014 & 0.8619 \\
Sample standard deviation & 0 & 0.2873 & 0.5746 & 100 \\
Number of Firms & 100 & 100 & 100 & 1,000 \\
Observations per firm & 1,000 & 1,000 & 1,000 &
\end{tabular}

PANEL B: Simulated regressions of filtered firm performance on noisy signal of common risk

\begin{tabular}{lrrrr}
\hline & $(1)$ & $(2)$ & $(3)$ & $(4)$ \\
\hline Risk exposure $\left(\hat{b}_{1}\right)$ & & & & \\
Mean & 0.0000 & 0.0001 & 0.0002 & 0.0002 \\
Standard deviation & 0.0032 & 0.0047 & 0.0078 & 0.0112 \\
Min & -0.0106 & -0.0156 & -0.0307 & -0.0458 \\
Max & 0.0099 & 0.0155 & 0.0238 & 0.0344 \\
t-values & & & & \\
$\quad$ Mean & -0.0039 & 0.0255 & 0.0318 & 0.0336 \\
Standard deviation & 1.0060 & 1.1523 & 1.2793 & 1.3286 \\
Share of $\hat{b}_{1}$-coefficients & & & & $13.90 \%$ \\
significant at 95\%-level & $5.80 \%$ & $9.30 \%$ & $12.70 \%$ & \\
R-squared & & & & 0.000018 \\
$\quad$ Mean & 0.000010 & 0.000013 & 0.000016 & 0.000025 \\
Standard deviation & 0.000014 & 0.000018 & 0.000023 & 1,000 \\
Observations & 1,000 & 1,000 & 1,000 & \\
\hline
\end{tabular}


Table 6: Simulation of pooled regression for a firm sample with proxy for exposure risk

PANEL A shows the relevant parameters used for simulating firm performance $\tilde{x}_{i t}=k+\tilde{c}_{i t} \cdot \tilde{\eta}_{t}+\tilde{\varepsilon}_{i t}$ and exposure to common risk $\tilde{\eta}_{t}$. For each regression a sample of 100 firms with 1,000 observations per firm were randomly generated using the parameter $k=1$ and independent random numbers generated from the distributions: $\tilde{\eta}_{t} \sim$ $\mathrm{N}(1,1), \tilde{c}_{i t} \sim \mathrm{N}\left(1, \sigma_{i}^{2}\right)$, and $\tilde{\varepsilon}_{i t} \sim \mathrm{N}(0,1)$. The firm specific standard deviations of the exposure to common risk, $\sigma_{i}$, was drawn once from a uniform distribution on $[0,2]$ as in regression model 3 in Table 5 at the beginning of the simulation study.

PANEL B presents the summary statistics of 1,000 estimations of the regression model $z_{i t}=b_{0}+b_{1} \cdot \eta_{t}+d+b_{2} \cdot d \cdot \eta_{t}+e_{i t}$ where $z_{i t}=x_{i t}-E\left[\tilde{c}_{i t}\right] \cdot \eta_{t}, E\left[\tilde{c}_{i t}\right]=1$, and $d$ is a dummy variable taking the value of 1 if the signal $\tilde{y}_{i}=\sigma_{i}+\tilde{\epsilon}$ takes a value above the sample mean of all signal realizations. The noise term in $\tilde{y}_{i}$ is randomly drawn from $\mathrm{N}\left(0, \sigma_{\epsilon}^{2}\right)$ with the standard deviations given in PANEL A.

PANEL A: Parameters used for the standard deviation of the noise term in the signal $\tilde{y}_{i}$

\begin{tabular}{rrrrr}
\hline & \multicolumn{3}{c}{ Regression model } & $(4)$ \\
\cline { 2 - 5 } & $(1)$ & $(2)$ & $(3)$ & 3
\end{tabular}

PANEL B: Simulated regressions of filtered firm performance on noisy signal of common risk

\begin{tabular}{|c|c|c|c|c|}
\hline & (1) & $(2)$ & $(3)$ & (4) \\
\hline \multicolumn{5}{|l|}{ Risk exposure $\left(\hat{b}_{1}\right)$} \\
\hline Mean & -0.0002 & 0.0008 & -0.0007 & -0.0003 \\
\hline Standard deviation & 0.0220 & 0.0297 & 0.0340 & 0.0339 \\
\hline Min & -0.0997 & -0.1032 & -0.1114 & -0.1085 \\
\hline Max & 0.0670 & 0.0985 & 0.1120 & 0.1015 \\
\hline \multicolumn{5}{|l|}{ t-values } \\
\hline Mean & -0.0090 & 0.0303 & -0.0237 & -0.0105 \\
\hline Standard deviation & 0.7984 & 1.0827 & 1.2440 & 1.2488 \\
\hline \multicolumn{5}{|l|}{$\begin{array}{l}\text { Risk exposure } \times \text { risk } \\
\text { exposure above mean }\left(\hat{b}_{2}\right)\end{array}$} \\
\hline Mean & 0.0001 & -0.0019 & 0.0008 & 0.0002 \\
\hline Standard deviation & 0.0501 & 0.0510 & 0.0528 & 0.0509 \\
\hline Min & -0.1590 & -0.1735 & -0.1712 & -0.1665 \\
\hline Max & 0.1769 & 0.1659 & 0.1806 & 0.1342 \\
\hline \multicolumn{5}{|l|}{ t-values } \\
\hline Mean & 0.0028 & -0.0512 & 0.0208 & 0.0046 \\
\hline Standard deviation & 1.2974 & 1.3143 & 1.3711 & 1.3260 \\
\hline $\begin{array}{l}\text { Share of } \hat{b}_{1} \text {-coefficients } \\
\text { significant at } 95 \% \text {-level }\end{array}$ & $1.10 \%$ & $6.90 \%$ & $11.20 \%$ & $11.80 \%$ \\
\hline $\begin{array}{l}\text { Share of } \hat{b}_{2} \text {-coefficients } \\
\text { significant at 95\%-level }\end{array}$ & $13.60 \%$ & $13.90 \%$ & $14.80 \%$ & $13.10 \%$ \\
\hline \multicolumn{5}{|l|}{ R-squared } \\
\hline Mean & 0.000452 & 0.000455 & 0.000468 & 0.000452 \\
\hline Standard deviation & 0.000488 & 0.000434 & 0.000450 & 0.000420 \\
\hline Observations & 1,000 & 1,000 & 1,000 & 1,000 \\
\hline
\end{tabular}


Table 7: Simulation of random coefficients regression for a firm sample

PANEL A shows the relevant parameters used for simulating firm performance $\tilde{x}_{i t}=k+\tilde{c}_{i t} \cdot \tilde{\eta}_{t}+\tilde{\varepsilon}_{i t}$ and exposure to common risk $\tilde{\eta}_{t}$. For each regression a sample of 100 firms with 1,000 observations per firm was randomly generated using the parameter $k=1$ and independent random numbers generated from the distributions $\tilde{\eta}_{t} \sim$ $\mathrm{N}(1,1), \tilde{c}_{i t} \sim \mathrm{N}\left(1, \sigma_{i}^{2}\right)$, and $\tilde{\varepsilon}_{i t} \sim \mathrm{N}(0,1)$. The firm specific standard deviations of the exposure to common risk, $\sigma_{i}$, were drawn once from the uniform distributions given in the first row of PANEL A at the beginning of the simulation study.

PANEL B presents the summary statistics of 1,000 estimations of the random coefficients regression model $z_{i t}=b_{0 i}+b_{1 i} \cdot \eta_{t}+e_{i t}$ where $b_{1 i}=b_{1}+v_{i}, z_{i t}=x_{i t}-E\left[\tilde{c}_{i t}\right] \cdot \eta_{t}$, and $E\left[\tilde{c}_{i t}\right]=1$.

PANEL A: Parameter distribution for the standard deviation of $\tilde{c}_{i t}$ used for simulating firm performance

\begin{tabular}{lrrrr}
\hline & \multicolumn{3}{c}{ Regression model } & $(4)$ \\
\cline { 2 - 5 } & $(1)$ & $(2)$ & $(3)$ & $\mathrm{U}[0,3]$ \\
Initial Distribution of $\sigma_{0}$ & - & $\mathrm{U}[0,1]$ & $\mathrm{U}[0,2]$ & 1.5021 \\
Sample Mean & 0 & 0.5007 & 1.0014 & 0.8619 \\
Sample standard deviation & 0 & 0.2873 & 0.5746 & 100 \\
Number of Firms & 100 & 100 & 100 & 1,000 \\
Observations per firm & 1,000 & 1,000 & 1,000 &
\end{tabular}

PANEL B: Simulated regressions of filtered firm performance on noisy signal of common risk

\begin{tabular}{|c|c|c|c|c|}
\hline & (1) & (2) & $(3)$ & (4) \\
\hline \multicolumn{5}{|l|}{$\begin{array}{l}\text { Common component of } \\
\text { risk exposure }\left(\hat{b}_{1}\right)\end{array}$} \\
\hline Mean & 0.0000 & 0.0001 & 0.0002 & 0.0003 \\
\hline Standard deviation & 0.0032 & 0.0045 & 0.0068 & 0.0094 \\
\hline Min & -0.0106 & -0.0149 & -0.0277 & -0.0405 \\
\hline Max & 0.0097 & 0.0142 & 0.0209 & 0.0293 \\
\hline \multicolumn{5}{|l|}{ z-values } \\
\hline Mean & -0.0025 & 0.0185 & 0.0214 & 0.0217 \\
\hline Standard deviation & 0.7121 & 0.7149 & 0.7023 & 0.6947 \\
\hline $\begin{array}{l}\text { Share of } \hat{b}_{1} \text {-coefficients } \\
\text { significant at } 95 \% \text {-level }\end{array}$ & $0.50 \%$ & $0.40 \%$ & $0.30 \%$ & $0.40 \%$ \\
\hline Observations & 1,000 & 1,000 & 1,000 & 1,000 \\
\hline
\end{tabular}

